$\underline{\text { Preprint typeset in JHEP style - HYPER VERSION }}$

hep-th/0407216

LPTHE-04-19

LPTENS-04-34

WIS/19/04-JUL-DPP

\title{
Closed Strings in Misner Space: Stringy Fuzziness with a Twist
}

\author{
M. Berkooz $\diamond^{*}$, B. Durin^, B. Pioline ${ }^{2 \uparrow}$, D. Reichmann $\diamond$ \\ $\diamond$ Weizmann Institute of Science, \\ Rehovot 76100, Israel \\ a LPTHE, Universités Paris 6 et 7, 4 place Jussieu, \\ 75252 Paris cedex 05, France \\ ه LPTENS, Département de Physique de l'ENS, 24 rue Lhomond, \\ 75231 Paris cedex 05, France \\ E-mail: \\ berkooz@wisemail.weizmann.ac.il, bdurin@lpthe.jussieu.fr, \\ pioline@lpthe.jussieu.fr, tragula@wisemail.weizmann.ac.il
}

ABStRaCt: Misner space, also known as the Lorentzian orbifold $\mathbb{R}^{1,1} /$ boost, is the simplest tree-level solution of string theory with a cosmological singularity. We compute tree-level scattering amplitudes involving twisted states, using operator and current algebra techniques. We find that, due to zero-point quantum fluctuations of the excited modes, twisted strings with a large winding number $w$ are fuzzy on a scale $\sqrt{\log w}$, which can be much larger than the string scale. Wave functions are smeared by an operator $\exp \left(\Delta(\nu) \partial_{+} \partial_{-}\right)$ reminiscent of the Moyal-product of non-commutative geometry, which, since $\Delta(\nu)$ is real, modulates the amplitude rather than the phase of the wave function, and is purely gravitational in its origin. We compute the scattering amplitude of two twisted states and one tachyon or graviton, and find a finite result. The scattering amplitude of two twisted and two untwisted states is found to diverge, due to the propagation of intermediate winding strings with vanishing boost momentum. The scattering amplitude of three twisted fields is computed by analytic continuation from three-point amplitudes of states with non-zero $p^{+}$ in the Nappi-Witten plane wave, and the non-locality of the three-point vertex is found to diverge for certain kinematical configurations. Our results for the three-point amplitudes allow in principle to compute, to leading order, the back-reaction on the metric due to a condensation of coherent winding strings.

*Incumbent of the Recanati career development chair for energy research 


\section{Contents}

1. Introduction 1

2. Review of the first quantization of twisted strings 3

2.1 The geometry of the orbifold

2.2 Quantization of the twisted sectors

2.3 Two-point function of twisted fields 6

2.4 Untwisted vertex operators and stringy fuzziness 8

2.4.1 Tachyon vertex operator 8

2.4 .2 Gauge boson and graviton vertex operators 9

2.5 Stringy fuzziness with a twist 10

3. Scattering amplitudes with two twisted strings 13

3.1 Two-twisted one-untwisted amplitude 14

3.1.1 Tachyon form factor 14

3.1.2 Graviton form factor 16

3.2 Two-twisted two-untwisted scattering amplitude 17

4. Scattering amplitudes with more than two twisted strings 20

4.1 Misner space and the Nappi-Witten plane wave 20

4.2 Three-twist amplitude $\quad 22$

A. Scattering amplitudes of untwisted strings 24

A.1 Four-graviton amplitude in type II superstring 24

A.2 Scattering amplitudes for wave-packets in Grant space 26

\section{Introduction}

Misner space [1], also known as the Lorentzian orbifold $\mathbb{R}^{1,1} /$ boost, is an especially attractive exact solution of string theory at tree-level [2]. Since it involves two cosmological (Milne) regions connected by a space-like singularity, it is an interesting toy model to study whether quantum effects may resolve cosmological singularities, and give ground for pre-Big Bang scenarios [3] (see [4, 5, 6, 7, 8, 9, 10] for other $\alpha^{\prime}$-exact models of cosmological singularities). Since it also involves two static (Rindler) regions with compact time attached at the singularity, it may also be a useful setting to discuss chronology protection in string theory. Finally, Misner's cousin Grant space [11], the orbifold $\mathbb{R}^{1,2} /($ boost $\times$ translation $)$, arises as a limit of the inside of BTZ black hole [12, 13], hence may have bearing on the fate of certain black hole singularities. 
Being a quotient of flat space, Misner space may be amenable to standard conformal theory techniques, properly extended to account for its Lorentzian signatures and non-compact nature [14. In particular, it includes both untwisted states, corresponding to gravitons and other particles propagating in the geometry, and an infinite number of twisted sectors, corresponding to strings winding around the compact, space-like or timelike, directions [15, 16]. While tree-level scattering amplitudes of untwisted states have been shown to lead to severe divergences [17, 18] (see [6, 7] for a study of scattering amplitudes in a related model), we have shown in 15, 16] that twisted strings, both in the Milne and Rindler regions, are pair-produced quantum-mechanically, and found indications that the resulting back-reaction may in fact resolve the singularity. While a consistent treatment of the back-reaction of correlated pairs of winding strings is still out of reach, a somewhat less ambitious approach is to marginally deform the orbifold CFT by a coherent superposition of twisted strings with a given winding number, and determine the deformed metric order by order in the conformal perturbation, if the true conformal field theory happens to be close enough from the orbifold point.

In this paper, we take another step in this direction, and study tree-level scattering of twisted and untwisted sector states of the bosonic string in Misner space. Amplitudes involving two twisted states can be computed by operator techniques, after expressing untwisted vertex operators in terms of the mode expansion in the twisted sector with winding number $w$. We carry this out in Section 2 below, and find that, due to the zero-point quantum fluctuations of the excited modes, the energy momentum and other untwisted sector currents sourced by a twisted string, are smeared over a characteristic distance $\sqrt{\Delta(\nu)}$, where $\Delta(\nu)$, displayed in (2.36) below, is the regulated r.m.s. size of the string in the twisted sector $w=-\nu / \beta$, where $\beta$ is the rapidity of the boost transformation. In particular, for large winding number $w$, the size grows as $\Delta(\nu) \sim \log w$, and can thus become arbitrarily larger than the string scale $\alpha^{\prime}$. This fuzziness is qualitatively similar to non-commutative geometry, in as much as sources are smeared by an exponential of the square of the momentum, $\exp \left[-p^{+} p^{-} \Delta(\nu)\right]$. The extended nature of the string can therefore manifest itself down to very low energies if $\Delta(\nu)$ is large enough. In contrast to the Moyal product however, this non-locality arises at the level of charged states rather than dipoles, and modulates the amplitude rather than the phase of the wave function. It arises purely in the closed string sector, through gravitational effects due to cosmological singularity. This non-locality implies that a condensation of long strings in the Rindler regions may have an important effect in the cosmological regions, and possibly resolve the singularity. It is tempting to speculate that such a non-locality may also be relevant for the dynamics of the BTZ black hole inside the horizon.

In Section 3, using this twisted mode representation for the untwisted vertex operators, we compute three-point amplitudes involving two twisted states in their "tachyonic" ground state (a non-vanishing transverse momentum can be chosen to ensure that the state is in fact not tachyonic in the two light-cone directions) and one off-shell tachyon or graviton. The result can be interpreted as the tachyon source or energy-momentum sourced by a single twisted string. Using a real space representation for the "quasi-zero-modes" of the string, and the relation to the charged particle problem discussed in [15, 16], we find that 
the amplitude is given by the overlap of the wave functions of two charged particles and one neutral particle, up to the smearing kernel discussed above. It is in particular finite. We proceed to study the 4-point amplitude of two twisted states and two untwisted ones (two tachyons, or one tachyon and one graviton), and find that the amplitude diverges whenever the intermediate winding string has zero boost-momentum: this appears to be related to a large emission of winding strings close to the cosmological singularity. This result parallels the discussion of the four-untwisted scattering amplitude in [17], which was found to diverge due to large graviton exchange near the singularity.

Finally, in Section 4, we turn to amplitudes involving more than two twisted states: in this case, operator methods are no longer applicable. Nevertheless, correlators of Lorentzian twist fields can be obtained by analytic continuation from those of Euclidean twist fields, i.e. fields creating a cut $\left(Z \rightarrow e^{2 \pi i \theta} Z, \bar{Z} \rightarrow e^{-2 \pi i \theta} \bar{Z}\right)$ on the Euclidean two-plane $(Z, \bar{Z})$, with an generally irrational angle $\theta$. Such twist fields occur as subfactors in the vertex operators of states with $p^{+}=\theta \neq 0$ in the Nappi-Witten plane wave, and their correlation functions have been computed recently using current algebra techniques [23, 24] or a Wakimoto-type representation [25, 26]. Using this correspondence, we compute the threepoint vertex of three twisted states, and find its real-space representation (4.16). Again, we find that the interaction vertex is spread over a distance $\Xi\left(\nu_{1}, \nu_{2}\right)$ given in (4.17). For special kinematical configurations, this non-locality scale becomes infinite: it would be interesting to understand the precise physics behind this singularity.

Using our results in Section 3 and 4, one may now compute the back-reaction on the tachyon and massless, twisted or untwisted fields, induced by the condensation of a single twisted string, to leading order in the deformation parameter. We leave this analysis to further work.

For completeness, scattering amplitudes of untwisted states are discussed in Appendix A, extending the analysis of [17, 6, 7] to the cases of gravitons in the bosonic string / superstring in Misner / Grant space. We find that, unlike the null brane, constructing wave-packets with continuous boost momentum does not help in removing divergences in Grant space, although it does reduce the range of dangerous momenta.

\section{Review of the first quantization of twisted strings}

\subsection{The geometry of the orbifold}

Recall that Misner space is defined as the orbifold of two-dimensional flat Minkowski space $\mathbb{R}^{1,1}$ by the discrete boost $\left(x^{+}, x^{-}\right) \rightarrow\left(e^{2 \pi \beta} x^{+}, e^{-2 \pi \beta} x^{-}\right)$. The geometry of the quotient consists of four Lorentzian cones touching at their apex. The regions $x^{+}<0, x^{-}<0(\mathrm{P})$ and $x^{+}>0, x^{-}>0(\mathrm{~F})$, often called Milne regions, are cosmological geometries with a shrinking spatial circle collapsing in a Big Crunch, and an expanding spatial circle which begins in a Big Bang, respectively. The regions $x^{+}>0, x^{-}<0(\mathrm{R})$ and $x^{+}<0, x^{-}>0$ $(\mathrm{L})$, often termed Rindler regions, are time-independent geometries with a compact time: indeed, the Killing vector generating the boost is space-like in the Milne regions, but becomes time-like in the Rindler regions. 
As in regular Euclidean orbifolds, the spectrum consists of two kinds of states - untwisted and twisted. Untwisted strings correspond to particle-like excitations which are invariant under the orbifold action; for non-tachyonic two-dimensional mass $\mu^{2}$, these states are mostly localized in the Milne regions, although their wave function extends to a distance $r \sim|j| / \mu$ in the Rindler regions, where the "boost momentum" $j$ is the quantized momentum along the compact spatial circle (or energy with respect to the compact Rindler time). The twisted states will be reviewed momentarily after we introduce some of the required machinery.

Our goal in this paper is to study the scattering of untwisted states off a twisted string, and identify the sources that the twisted modes create for the untwisted graviton and tachyon. A effect that we will discuss depends crucially on the a correct treatment of both the excited and the quasi-zero modes of the string, which we will now survey.

\subsection{Quantization of the twisted sectors}

Closed strings in the $w$-th twisted sector of the Lorentzian satisfy the twisted periodicity condition around the cylinder,

$$
X^{ \pm}(\sigma+2 \pi, \tau)=e^{ \pm 2 \pi w \beta} X^{ \pm}(\sigma, \tau)
$$

The embedding coordinates $X^{ \pm}$thus admit the normal mode expansion (in the units where $\left.\alpha^{\prime}=2\right)$

$$
X^{ \pm}(\tau, \sigma)=i \sum_{n=-\infty}^{\infty}(n \pm i \nu)^{-1} \alpha_{n}^{ \pm} e^{-i(n \pm i \nu)(\tau-\sigma)}+i \sum_{n=-\infty}^{\infty}(n \mp i \nu)^{-1} \tilde{\alpha}_{n}^{ \pm} e^{-i(n \mp i \nu)(\tau+\sigma)}
$$

where the imaginary part of the frequency is related to the winding number and boost parameter by $\nu=-w \beta$. The coordinates $\tau, \sigma$ denote the (non-compact) time and (compact) spatial directions of the Lorentzian world-sheet, and are related to the Euclidean coordinates of radial quantization by $z=e^{i(\tau-\sigma)}, \bar{z}=e^{i(\tau+\sigma)}$.

As for strings in flat space, left and right-moving excitations are decoupled, and satisfy the following commutation relations and hermiticity properties,

$$
\begin{aligned}
{\left[\alpha_{m}^{+}, \alpha_{n}^{-}\right]=-(m+i \nu) \delta_{m+n} } & , \quad\left[\tilde{\alpha}_{m}^{+}, \tilde{\alpha}_{n}^{-}\right]=-(m-i \nu) \delta_{m+n} \\
\left(\alpha_{-n}^{ \pm}\right)^{*}=\alpha_{n}^{ \pm}, & \left(\tilde{\alpha}_{-n}^{ \pm}\right)^{*}=\tilde{\alpha}_{n}^{ \pm}
\end{aligned}
$$

Of particular interest are the (quasi) zero-modes $\alpha_{0}^{ \pm}$and $\tilde{\alpha}_{0}^{ \pm}$, which are self-adjoint and satisfy

$$
\left[\alpha^{+}, \alpha^{-}\right]=-i \nu, \quad\left[\tilde{\alpha}^{+}, \tilde{\alpha}^{-}\right]=i \nu
$$

where we dropped the subscript 0 for conciseness. Excited modes may be quantized just as in flat space, as creation and annihilation operators in a Fock space with ground state annihilated by $\alpha_{m>0}^{ \pm}$. In contrast, due to their unusual hermiticity properties, zero-modes require a particular treatment. They may be represented as covariant derivatives for a charged scalar field in an electric field [15],

$$
\alpha^{ \pm}=i \nabla_{\mp}=i \partial_{\mp} \pm \frac{\nu}{2} x^{ \pm}, \quad \tilde{\alpha}^{ \pm}=i \tilde{\nabla}_{\mp}=i \partial_{\mp} \mp \frac{\nu}{2} x^{ \pm}
$$


which ensures the proper hermiticity properties under the inner product

$$
\left\langle f_{1} \mid f_{2}\right\rangle=\int d x^{+} d x^{-} f_{1}^{*}\left(x^{+}, x^{-}\right) f_{2}\left(x^{+}, x^{-}\right)
$$

On-shell wave functions satisfying the physical state conditions $L_{0}=\bar{L}_{0}=1$ are thus eigenmodes of the Klein-Gordon operator for a charged particle in an electric field,

$$
\left(\alpha_{0}^{+} \alpha_{0}^{-}+\alpha_{0}^{-} \alpha_{0}^{+}\right) f=M^{2} f, \quad\left(\tilde{\alpha}_{0}^{+} \tilde{\alpha}_{0}^{-}+\tilde{\alpha}_{0}^{-} \tilde{\alpha}_{0}^{+}\right) f=\tilde{M}^{2} f
$$

where $M^{2}$ and $\tilde{M}^{2}$ are the contributions from the left and right moving excited modes. The orbital boost momentum $j$, generating boost transformations for the zero-modes, is given by

$$
j \equiv i\left(x^{+} \partial_{+}-x^{-} \partial_{-}\right)=\left(M^{2}-\tilde{M}^{2}\right) /(2 \nu)
$$

and fixed by the level matching condition $L_{0}-\bar{L}_{0}=0$. It is furthermore quantized in integer multiples of $1 / \beta$, in order for the twisted state to be invariant under the orbifold projection. It will be useful to further define the two-dimensional square mass $\mu^{2}=\left(M^{2}+\tilde{M}^{2}\right) / 2$, which, unlike $j$, takes continuous values.

The coordinate $x^{+}$introduced in (2.6) describes the location of the winding string in the Heisenberg representation (i.e. at $\tau=0)$, since the zero-mode part of $X^{ \pm}(\sigma, \tau)$ may be written as

$$
X_{0}^{ \pm}(\sigma, \tau)=e^{\mp \nu \sigma}\left[\cosh (\nu \tau) x^{ \pm} \pm \frac{2 i}{\nu} \sinh (\nu \tau) \partial_{\mp}\right]
$$

Notice that the dependence of the zero-mode on the $\sigma$ coordinate amounts to a boost in the $\left(x^{+}, x^{-}\right)$plane, i.e. a translation along the compact coordinate of the orbifold.

Alternatively, it is possible to use a representation where half of the covariant derivative operators are diagonalized, e.g.

$$
\alpha^{-}=i \nu \partial_{\alpha^{+}}, \quad \tilde{\alpha}^{+}=i \nu \partial_{\tilde{\alpha}^{-}}
$$

acting on functions of the variables $\alpha^{+}, \tilde{\alpha}^{-}$taking values in the quadrant $\mathbb{R}^{\epsilon} \times \mathbb{R}^{\tilde{\epsilon}}$. On-shell wave functions are now powers of their arguments,

$$
f\left(\alpha^{+}, \tilde{\alpha}^{-}\right)=N_{i n}\left(\epsilon \alpha^{+}\right)^{\frac{M^{2}}{2 i \nu}-\frac{1}{2}}\left(\tilde{\epsilon} \tilde{\alpha}^{-}\right)^{\frac{\tilde{M}^{2}}{2 i \nu}-\frac{1}{2}}
$$

The notation $N_{\text {in }}$ for the normalization factor, to be computed below, anticipates the fact that this representation is appropriate to describe an in state. This representation is the analogue of the oscillator representation for the standard harmonic oscillator, however, since the powers are not integers, it involves four different branches, according to the signs $\epsilon$ and $\tilde{\epsilon}$ of $\alpha^{-}$and $\tilde{\alpha}^{+}$. These gives rise to qualitatively distinct classes of twisted sector states:

- Short strings: for $\epsilon \tilde{\epsilon}=1$ the strings wind around the Milne spatial circle, and propagate from infinite past to infinite future. When $j \neq 0$, they also extend in the Rindler regions to a finite distance $r_{-}^{2}=(M-\tilde{M})^{2} /\left(4 \nu^{2}\right)$. 
- Long strings: for $\epsilon \tilde{\epsilon}=-1$, the strings instead live in the Rindler regions only, and correspond to static configurations which extend from spatial infinity in L or R to a finite distance $r_{+}^{2}=(M+\tilde{M})^{2} /\left(4 \nu^{2}\right)$, and folding back to infinity again. In addition, there exists tunneling configurations whereby a long string dissolves at a distance $r_{+}$ and reemerges as a short string at distance $r_{-}$, before escaping into the cosmological regions $(\mathrm{P}$ and $\mathrm{F})$.

In particular, in contrast to Euclidean orbifolds, twisted strings are in no sense localized near the singularity.

The oscillator representation (2.11) can be related to the real-space representation (2.6) via

$$
f\left(x^{+}, x^{-}\right)=\int d \tilde{\alpha}^{+} d \alpha^{-} \Phi_{\nu, \tilde{\alpha}^{+}, \alpha^{-}}^{i n}\left(x^{+}, x^{-}\right) f\left(\alpha^{+}, \tilde{\alpha}^{-}\right)
$$

where the kernel is given by

$$
\Phi_{\nu, \alpha^{+}, \tilde{\alpha}^{-}}^{i n}\left(x^{+}, x^{-}\right)=\exp \left(\frac{i \nu x^{+} x^{-}}{2}-i \alpha^{+} x^{-}-i \tilde{\alpha}^{-} x^{+}+\frac{i}{\nu} \alpha^{+} \tilde{\alpha}^{-}\right)
$$

This kernel may be viewed as the wave function for an off-shell winding state with momenta $\alpha^{+}$and $\tilde{\alpha}^{-}$. Equivalently, one may diagonalize the complementary set of operators,

$$
\alpha^{+}=-i \nu \partial_{\alpha^{-}}, \quad \tilde{\alpha}^{-}=-i \nu \partial_{\tilde{\alpha}^{+}}
$$

leading to on-shell wave functions

$$
f\left(\alpha^{-}, \tilde{\alpha}^{+}\right)=N_{\text {out }}\left(\epsilon \alpha^{-}\right)^{-\frac{M^{2}}{2 i \nu}-\frac{1}{2}}\left(\tilde{\epsilon} \tilde{\alpha}^{+}\right)^{-\frac{\tilde{M}^{2}}{2 i \nu}-\frac{1}{2}}
$$

Those are related to the real-space representation by the kernel

$$
\Phi_{\nu, \tilde{\alpha}^{+}, \alpha^{-}}^{\text {out }}\left(x^{+}, x^{-}\right)=\exp \left(-\frac{i \nu x^{+} x^{-}}{2}-i \tilde{\alpha}^{+} x^{-}-i \alpha^{-} x^{+}-\frac{i}{\nu} \tilde{\alpha}^{+} \alpha^{-}\right)
$$

\subsection{Two-point function of twisted fields}

As usual, the two-point function of twisted fields vanishes unless the two fields carry the same winding number. In addition, since the excited modes are quantized as in flat space, the amplitude is non-zero only when the excitation levels on the left and on the rightmoving side are equal. Throughout this section we will limit ourselves to the case of twisted strings without internal excitation, but with some internal momentum $k_{i}$ so that the resulting state is non-tachyonic in 2 dimensions. For generality however, we will not use any specific value for the two-dimensional masses $M^{2}$ and $\tilde{M}^{2}$.

We will find it convenient to use the representations (2.12) and (2.16) (or rather, its hermitian conjugate) for the in and out twisted states, respectively. The inner product between in and out states may be simply determined by returning to the real-space representation and using (2.7):

$$
{ }_{\text {out }}\left\langle f_{1} \mid f_{2}\right\rangle_{\text {in }}=\int d \tilde{\alpha}^{+} d \alpha^{-} d \alpha^{+} d \tilde{\alpha}^{-} f_{1}^{*}\left(\tilde{\alpha}^{+}, \alpha^{-}\right) e^{\frac{i}{\nu}\left(\alpha^{+} \alpha^{-}+\tilde{\alpha}^{+} \tilde{\alpha}^{-}\right)} f_{2}\left(\alpha^{+}, \tilde{\alpha}^{-}\right)
$$


where the integration domain is $\mathbb{R}^{\epsilon_{1}} \times \mathbb{R}^{\tilde{\epsilon}_{1}} \times \mathbb{R}^{\epsilon_{2}} \times \mathbb{R}^{\tilde{\epsilon}_{2}}$. The overlap may be computed straightforwardly in terms of Gamma functions, and yields

$$
\begin{aligned}
{ }_{\text {out }}\left\langle f_{1} \mid f_{2}\right\rangle_{\text {in }}= & N_{\text {out }}^{*} N_{\text {in }}\left[2 i \nu^{2}\left(i \nu \epsilon_{1} \epsilon_{2}\right)^{\frac{M_{2}^{2}}{2 i \nu}-\frac{1}{2}} \Gamma\left(\frac{M_{2}^{2}}{2 i \nu}+\frac{1}{2}\right) \delta\left(M_{1}^{2}-M_{2}^{2}\right)\right] \\
& \times\left[2 i \nu^{2}\left(i \nu \tilde{\epsilon}_{1} \tilde{\epsilon}_{2}\right)^{\frac{\tilde{M}_{1}^{2}}{2 i \nu}-\frac{1}{2}} \Gamma\left(\frac{\tilde{M}_{1}^{2}}{2 i \nu}+\frac{1}{2}\right) \delta\left(\tilde{M}_{1}^{2}-\tilde{M}_{2}^{2}\right)\right]
\end{aligned}
$$

where the first bracket corresponds to the $\left(\alpha^{+}, \alpha^{-}\right)$integral and the second to the $\left(\tilde{\alpha}^{+}, \tilde{\alpha}^{-}\right)$ integral. Due to the orbifold projection however, the momentum $j=\left(M^{2}-\tilde{M}^{2}\right) /(2 \nu)$ is a discrete quantity, hence its conservation should be enforced by a Kronecker symbol rather than Dirac's delta function. The way to remedy this problem is to mod out the integration domain on $\left(\alpha^{ \pm}, \tilde{\alpha}^{ \pm}\right)$by the same boost which identified the $x^{ \pm}$coordinates,

$$
\left(\alpha^{ \pm}, \tilde{\alpha}^{ \pm}\right) \equiv\left(e^{ \pm 2 \pi \beta} \alpha^{ \pm}, e^{ \pm 2 \pi \beta} \tilde{\alpha}^{ \pm}\right)
$$

This action clearly leaves the on-shell wave functions with quantized $j(2.12),(2.16)$ invariant, and is consistent with the kernels $(2.14), 2.17)^{1}$. Under this prescription, and upon choosing the normalization factors

$$
N_{\text {in }}=N_{\text {out }}^{*}=(2 \nu)^{-1 / 2}(i \nu)^{-\frac{M^{2}+\tilde{M}^{2}}{4 i \nu}-\frac{1}{2}}\left[\Gamma\left(\frac{M^{2}}{2 i \nu}+\frac{1}{2}\right) \Gamma\left(\frac{\tilde{M}^{2}}{2 i \nu}+\frac{1}{2}\right)\right]^{-1 / 2}
$$

we obtained delta-normalized states,

$$
{ }_{\text {out }}\left\langle f_{1} \mid f_{2}\right\rangle_{\text {in }}=\delta\left(\mu_{1}^{2}-\mu_{2}^{2}\right) \delta_{j_{2}-j_{1}}\left(\epsilon_{1} \epsilon_{2}\right)^{\frac{M_{2}^{2}}{2 i \nu}-\frac{1}{2}}\left(\tilde{\epsilon}_{1} \tilde{\epsilon}_{2}\right)^{\frac{\tilde{M}_{1}^{2}}{2 i \nu}-\frac{1}{2}}
$$

For simplicity, we will consider only amplitudes involving the same type of twisted states for the in and out states $\epsilon_{1}=\epsilon_{2}$ and $\tilde{\epsilon}_{1}=\tilde{\epsilon}_{2}$.

Finally, for the purpose of computing more general overlaps, it will be useful to use generating function techniques, and view the on-shell in wave function (2.12) as a derivative w.r.t. generating parameters $\left(\zeta^{-}, \tilde{\zeta}^{+}\right)$with continuous order,

$$
f\left(\alpha^{+}, \tilde{\alpha}^{-}\right)=\left.\mathcal{D}_{M^{2}, \epsilon, \tilde{\epsilon}}^{i n} \cdot e^{-i \alpha^{+} \zeta^{-}-i \tilde{\alpha}^{-} \tilde{\zeta}^{+}}\right|_{\zeta^{-}=\tilde{\zeta}^{+}=0}
$$

where

$$
\mathcal{D}_{M^{2}, \epsilon, \tilde{\epsilon}}^{i n} \equiv N_{i n}\left[i \epsilon \frac{\partial}{\partial \zeta^{-}}\right]^{\frac{M^{2}}{2 i \nu}-\frac{1}{2}}\left[i \tilde{\epsilon} \frac{\partial}{\partial \tilde{\zeta}^{+}}\right]^{\frac{\tilde{M}^{2}}{2 i \nu}-\frac{1}{2}}
$$

In this expression, the continuous derivative operator is defined by ${ }^{2}$

$$
\left.\frac{\partial^{\lambda}}{\partial x^{\lambda}} f(x)\right|_{x=0}=\frac{(-1)^{\lambda}}{\Gamma(-\lambda)} \int_{0}^{\infty} d x x^{-\lambda-1} f(x),
$$

\footnotetext{
${ }^{1}$ It may seem that one should mod out the $\alpha^{ \pm}$and $\tilde{\alpha}^{ \pm}$planes by independent boosts, however this would lead to a conservation of both $M^{2}$ and $\tilde{M}^{2}$ by Kronecker symbols, inconsistent with the continuous nature of $\mu^{2}$.

${ }^{2}$ E.g, acting on $f(x)=e^{a x}$ yields $a^{\lambda}$ as it should.
} 
Similarly, the out wave function may be represented as a derivative with respect to a second set of parameters $\left(\zeta^{+}, \tilde{\zeta}^{-}\right)$,

$$
f\left(\tilde{\alpha}^{+}, \alpha^{-}\right)=\left.\mathcal{D}_{M^{2}, \epsilon, \tilde{\epsilon}}^{\text {out }} \cdot e^{-i \tilde{\alpha}^{+} \tilde{\zeta}^{-}-i \alpha^{-} \zeta^{+}}\right|_{\zeta^{+}=\tilde{\zeta}^{-}=0}
$$

where

$$
\mathcal{D}_{M^{2}, \epsilon, \tilde{\epsilon}}^{\text {out }} \equiv N_{\text {out }}\left[i \tilde{\epsilon} \frac{\partial}{\partial \tilde{\zeta}^{-}}\right]^{-\frac{\tilde{M}^{2}}{2 i \nu}-\frac{1}{2}}\left[i \epsilon \frac{\partial}{\partial \zeta^{+}}\right]^{-\frac{M^{2}}{2 i \nu}-\frac{1}{2}}
$$

For simplicity, we will drop the indices from the operators $\mathcal{D}$ when no confusion is possible. The integral (2.18) over $\alpha^{ \pm}, \tilde{\alpha}^{ \pm}$is now Gaussian ${ }^{3}$,

$$
\left\langle f_{1} \mid f_{2}\right\rangle=\left.\nu^{2} \mathcal{D}_{1}^{*} \mathcal{D}_{2} \cdot e^{i \nu\left(\zeta^{+} \zeta^{-}+\tilde{\zeta}^{+} \tilde{\zeta}^{-}\right)}\right|_{\zeta^{ \pm}=\tilde{\zeta}^{ \pm}=0}
$$

and the overlap (2.19) follows from the formal derivation rule

$$
\left.\left[i \epsilon_{1} \frac{\partial}{\partial \zeta^{+}}\right]^{\frac{M_{1}^{2}}{2 i \nu}-\frac{1}{2}}\left[i \epsilon_{2} \frac{\partial}{\partial \zeta^{-}}\right]^{\frac{M_{2}^{2}}{2 i \nu}-\frac{1}{2}} e^{i \nu \zeta^{+} \zeta^{-}}\right|_{\zeta^{ \pm}=0}=2 i \nu^{2}\left(i \nu \epsilon_{1} \epsilon_{2}\right)^{\frac{M_{2}^{2}}{2 i \nu}-\frac{1}{2}} \Gamma\left(\frac{M_{2}^{2}}{2 i \nu}+\frac{1}{2}\right) \delta\left(M_{1}^{2}-M_{2}^{2}\right)
$$

which generalizes to continuous order the familiar fact for ordinary (integer order) derivatives,

$$
\left.\left[i \frac{\partial}{\partial \zeta^{+}}\right]^{n}\left[i \frac{\partial}{\partial \zeta^{-}}\right]^{m} e^{i \nu \zeta^{+} \zeta^{-}}\right|_{\zeta^{ \pm}=0}=(i \nu)^{n} \Gamma(n+1) \delta_{m-n}
$$

Notice that the "generating" $\left(\zeta^{+}, \tilde{\zeta}^{-}\right)$representation is simply obtained by Fourier transform from the $\left(\alpha^{-}, \tilde{\alpha}^{+}\right)$oscillator representation, hence is equivalent to the $\left(\alpha^{+}, \tilde{\alpha}^{-}\right)$, up to a rescaling by $\nu$.

\subsection{Untwisted vertex operators and stringy fuzziness}

Tree-level scattering amplitudes involving two twisted states and an arbitrary number of untwisted states can be computed by operator methods, by choosing a Lorentzian worldsheet which is an infinite cylinder, in the in and out twisted vacua at $\tau= \pm \infty$. For this, it is important to evaluate the action of untwisted vertex operators on the twisted Hilbert space.

\subsubsection{Tachyon vertex operator}

The tachyon vertex operator may be defined in the untwisted sector by the normal ordered expression

$$
V_{T}=: e^{i k^{+} X^{-}+k^{-} X^{+}}:(z) \equiv \lim _{w \rightarrow z} e^{i k^{+} X^{-}}(w) e^{i k^{-} X^{+}}(z) e^{k^{+} k^{-}\left[X_{>0}^{-}(z), X_{<0}^{+}(w)\right]}
$$

where $X_{\gtrless}^{ \pm}$denotes the positive and negative frequency parts of $X^{ \pm}$, as defined by the untwisted mode expansion. Notice that the operators $e^{i k^{ \pm} X^{\mp}}$ do not need any normal ordering prescription as there are no short distance singularities between $X^{ \pm}$and itself. In

\footnotetext{
${ }^{3}$ The peculiar integration domain does not make any difference for this argument.
} 
order to obtain a state invariant under the orbifold action, it is necessary to further smear (2.31) over the action of the boost $k^{ \pm} \rightarrow e^{ \pm v} k^{ \pm}$, but we will refrain from explicitly doing so in order to avoid cluttering. The definition above is the same as the familiar CFT normal ordering definition

$$
V_{T} \equiv \lim _{w \rightarrow z} e^{i k^{+} X^{-}}(w) e^{i k^{-} X^{+}}(z) e^{k^{+} k^{-} \log (w-z)}
$$

which we can then easily transfer to other sectors.

In terms of the twisted mode expansion, the normal ordered (with respect to the twisted sector vacuum) tachyon vertex operator $V_{T}$ now reads

$$
V_{T}=e^{i\left(k^{+} X_{\prec 0}^{-}+k^{-} X_{\prec 0}^{+}\right)} e^{i\left(k^{+} X_{\succ 0}^{-}+k^{-} X_{\succ 0}^{+}\right)} e^{i\left(k^{+} X_{0}^{-}+k^{-} X_{0}^{+}\right)} e^{k^{+} k^{-}\left(\left[X_{\succ 0}^{-}, X_{\succ 0}^{+}\right]-\left[X_{\succ 0}^{-}, X_{\prec 0}^{+}\right]\right)}
$$

where we have separated the the $X^{\prime}$ 's into positive and negative frequency parts $X_{\succ, \prec}^{ \pm}$of $X^{ \pm}$as defined by the twisted mode expansion. $X_{0}^{ \pm}$denotes the twisted quasi-zero-mode as in (2.10). The difference of commutators is now finite as $w \rightarrow z$, and evaluates to

$$
\Delta(\nu) \equiv\left[X_{\succ 0}^{-}, X_{\prec 0}^{+}\right]-\left[X_{>0}^{-}, X_{<0}^{+}\right]=\sum_{n>0}\left(\frac{2}{n}-\frac{1}{n+i \nu}-\frac{1}{n-i \nu}\right)
$$

The infinite sum can be evaluated in terms of the digamma function $\psi(x)=d \log \Gamma(x) / d x$,

$$
\Delta(\nu)=\psi(1+i \nu)+\psi(1-i \nu)-2 \psi(1)
$$

In particular, this implies that the excited states contribute a non-trivial form factor ${ }^{4}$ for untwisted strings in the background of a twisted string:

$$
\left\langle\nu\left|: e^{i\left(k^{+} X_{e x}^{-}+k^{-} X_{e x}^{+}\right)}:\right| \nu\right\rangle=\exp \left(-k^{+} k^{-} \Delta(\nu)\right)
$$

Notice that this result is unaffected by the projection on boost invariant states. We will return to the important implications of this form factor in Section 2.5 below, after discussing the graviton vertex operator.

\subsubsection{Gauge boson and graviton vertex operators}

We now turn to the gauge boson vertex operator; while this state does not satisfy the closed string level matching condition, it is a useful warm-up before tackling the graviton.

In the untwisted sector, the gauge boson vertex operator may be defined in terms of the tachyon vertex, as $^{5}$

$$
V_{A}^{\mu}=: z \partial_{z} X^{\mu} e^{i k X}: \equiv \lim _{w \rightarrow z} \frac{1}{2}\left[z \partial_{z} X^{\mu}(z) V_{T}(w)+V_{T}(z) w \partial_{w} X^{\mu}(w)\right]
$$

This differs from the more standard definition

$$
V_{A}^{\prime \mu} \equiv \lim _{w \rightarrow z} \frac{1}{2}\left[z \partial_{z} X^{\mu}(z) V_{T}(w)-\frac{i z k^{ \pm}}{z-w}\right]
$$

\footnotetext{
${ }^{4}$ Form factors in usual $Z_{N}$ Euclidean orbifolds have been discussed in Ref. 27]

${ }^{5}$ Recall that operators are implicitly radially ordered.
} 
by a total derivative term proportional to $k^{ \pm} \partial_{z} V_{T}$, and leads to the same results when restricting to physical polarizations $\zeta_{\mu}$ such that $\zeta_{\mu} k^{\mu}=0$. Nevertheless, the symmetric ordering prescription (2.37) has the advantage that the vertex operator for a longitudinal gauge boson becomes a total derivative, ${ }^{6}$

$$
i k_{\mu} V_{A}^{\mu}=z \partial_{z}: e^{i k X}:
$$

implying current conservation. While the non-conserved part of an electromagnetic current current does not source photons in the Lorentz gauge $\partial_{\mu} A^{\mu}=0$, we find it more convenient to maintain current conservation throughout.

Expressing $V_{A}$ in terms of the twisted mode expansion, we find the normal ordered expression

$$
V_{A}^{ \pm}=-i \sum_{n>0}\left[\alpha_{-n}^{ \pm} V_{T} z^{n}+V_{T} \alpha_{n}^{ \pm} z^{-n}\right] z^{\mp i \nu}-\frac{i}{2}\left[\alpha_{0}^{ \pm} V_{T}+V_{T} \alpha_{0}^{ \pm}\right] z^{\mp i \nu} \mp \nu k^{ \pm} V_{T}
$$

where the last term comes from normal ordering the excited modes of $\partial_{z} X^{ \pm}$through $V_{T}$. Notice that it amounts to shifting $\alpha_{0}^{ \pm} \rightarrow \alpha_{0}^{ \pm} \pm i \nu k^{ \pm} z^{ \pm i \nu}$. Similarly, computing the anti-holomorphic derivative one finds a shifting $\tilde{\alpha}_{0}^{ \pm} \rightarrow \tilde{\alpha}_{0}^{ \pm} \mp i \nu k^{ \pm} z^{ \pm i \nu}$. Importantly, the zero-mode part of the vertex operator appears to be symmetrized, as a requirement from current conservation.

Having dealt with the gauge boson, we can now obtain the vertex operator for the graviton, dilaton and Kalb-Ramond two-form by applying the same procedure on the holomorphic and anti-holomorphic sides:

$$
\begin{aligned}
V_{G}^{\mu \nu}= & : z \partial_{z} X^{\mu} \bar{z} \partial_{\bar{z}} X^{\nu} e^{i k X}: \\
\equiv & \lim _{w \rightarrow z, \bar{w} \rightarrow \bar{z}} \frac{1}{4}\left[z \partial_{z} X^{\mu} \bar{z} \partial_{\bar{z}} X^{\nu} V_{T}(w, \bar{w})+V_{T}(z, \bar{z}) w \partial_{w} X^{\mu}(w) \bar{w} \partial_{\bar{w}} X^{\nu}\right. \\
& \left.+z \partial_{z} X^{\mu} V_{T}(w, \bar{z}) \bar{w} \partial_{\bar{w}} X^{\nu}+\bar{z} \partial_{\bar{z}} X^{\nu} V_{T}(z, \bar{w}) w \partial_{w} X^{\mu}\right]
\end{aligned}
$$

Notice in the above expression that we radially ordered independently on the holomorphic and anti-holomorphic sides, and did not symmetrize in the polarization indices $(\mu, \nu)$ : the dilaton, graviton and Kalb-Ramond vertex operators can be obtained by extracting the trace from the ten-dimensional polarization tensor $\zeta_{\mu \nu}$ and (anti)symmetrizing. Inserting the twisted mode expansion, we obtain a normal ordered expression with respect to the positive/negative frequency excited twisted modes, while the quasi-zero-modes are symmetrized and shifted according to

$$
\alpha_{0}^{ \pm} \rightarrow \alpha_{0}^{ \pm} \pm i \nu k^{ \pm} z^{ \pm i \nu}, \quad \tilde{\alpha}_{0}^{ \pm} \rightarrow \tilde{\alpha}_{0}^{ \pm} \mp i \nu k^{ \pm} z^{ \pm i \nu}
$$

The energy-momentum tensor is then by construction conserved.

\subsection{Stringy fuzziness with a twist}

The form factor (2.36) appears in fact generically for all untwisted vertex operators, as a result of normal ordering in the twisted vacuum, and has important physical consequences, as we now explain.

\footnotetext{
${ }^{6}$ Checking this relation requires a careful handling of the zero-modes.
} 
It is of course well-known that, due to zero-point fluctuations, off-shell strings have a logarithmically diverging r.m.s. size [19]: for a scattering process of energy $E$, strings grow as large as $\sqrt{\log E}$, implying the standard Regge behavior $A \sim s^{t} \sim e^{t \log s}$. These divergences are usually absorbed by non-local wave function renormalization (i.e. normal ordering of the vertex operators), leading to local $n$-point functions at low energy.

In contrast, in the Lorentzian orbifold case, we find that zero-point fluctuations have a non-trivial dependence on the winding sector $w$, and therefore cannot be reabsorbed in a field redefinition of the untwisted states (nor can it be absorbed into a simple redefinition of the twisted states). Instead, as apparent from (2.36), the presence of a winding string polarizes ${ }^{7}$ untwisted string states into a cloud of r.m.s. size $\sqrt{\Delta(\nu)}$. This size is proportional to that of the winding string at small $\nu$, at large winding number however it grows logarithmically with $\nu$ :

$$
\begin{aligned}
\Delta(\nu) & =2 \zeta(3) \nu^{2}+O\left(\nu^{4}\right) \\
& =2 \log \nu-\frac{23}{20}+O\left(\nu^{-2}\right) .
\end{aligned}
$$

Importantly, in contrast to usual finite order Euclidean rotation orbifolds, the winding number is unbounded, and can lead to a fuzziness much larger than the string scale.

While the complete form factor depends on the zero-mode contributions as well as the excitation levels, it is instructive to consider the effect of the universal exponential factor (2.36) in real space. Since (2.36) diverges exponentially in the space-like region $k^{+} k^{-}<0$, it is necessary to assume that the remaining contribution $A_{0}\left(k^{+}, k^{-}\right)$of zero-modes and oscillators is sufficiently suppressed in this region. If so, the effect of (2.36) is to convolute $A_{0}\left(x^{+}, x^{-}\right)$with the Fourier transform of (2.36),

$$
A(y)=\int d^{2} y \exp \left(-\frac{\left(y^{+}-x^{+}\right)\left(y^{-}-x^{-}\right)}{\Delta(\nu)}\right) A_{0}(x)
$$

or, equivalently, to apply the non-local differential operator

$$
A(x)=\exp \left(\Delta(\nu) \partial_{+} \partial_{-}\right) A_{0}(x)
$$

This non-locality is reminiscent of non-commutative star products, but also markedly different, as it affects the amplitude rather than the phase of the wave function, and is already non-trivial at the level of single particle states. The analogy to the charged open string[15] makes it clear that this non-locality is to charged particles in an electric field what the non-commutative Moyal product is to neutral dipoles in a magnetic field. The important point however is that the "non-commutativity" arises here in the closed string sector, in the vicinity of a cosmological singularity. It would be interesting to develop a semi-classical picture of this non-locality, in analogy with the rigid dipole picture for the standard non-commutativity in a magnetic field [20].

\footnotetext{
${ }^{7}$ The same polarization effect takes place for charged open strings in an electric field, due to the analogy noted in 15$]$.
} 
The simplest example is a Gaussian distribution peaked at $\left(x_{0}^{+}, x_{0}^{-}\right)$with r.m.s. size $a$,

$$
A_{0}(x)=\exp \left(-\frac{\left(x^{+}-x_{0}^{+}\right)^{2}+\left(x^{-}-x_{0}^{-}\right)^{2}}{a^{2}}\right)
$$

whose transform $A(x)$ is

$$
\frac{a^{2}}{\sqrt{a^{4}-4 \Delta^{2}}} \exp \left(-\frac{a^{2}}{a^{4}-4 \Delta^{2}}\left[\left(x^{+}-x_{0}^{+}\right)^{2}+\left(x^{-}-x_{0}^{-}\right)^{2}+\frac{4 \Delta}{a^{2}}\left(x^{+}-x_{0}^{+}\right)\left(x^{-}-x_{0}^{-}\right)\right]\right) \text {, }
$$

The latter is still localized around $x_{0}^{ \pm}$, but deformed into an ellipse. The eigenvalues of the quadratic form are $1 /\left(a^{2}-2 \Delta\right)$ and $1 /\left(a^{2}+2 \Delta\right)$ and the main effect is to squeeze the r.m.s. fluctuations in one direction to zero as $\Delta$ approaches $a^{2} / 2$ (the direction that shrinks to zero eventually is parallel to $\left(x^{+}, x^{-}\right) \propto(1,-1)$ for $\left.\Delta>0\right)$.

Another example, more relevant for boost invariant situations, is the test function

$$
A_{0}\left(x^{+}, x^{-}\right)=\exp \left(-\frac{\left(x^{+} x^{-}+r_{0}^{2}\right)^{2}}{a^{2} r_{0}^{2}}\right)
$$

which, for real $r_{0}$, is localized around a finite distance $r_{0}$ in the Rindler wedges, with r.m.s size $a$. This can viewed as a slice of a "long string", localized around $r_{0}$ and winding around the compact Rindler time (for $r_{0}^{2}<0$, it instead describes a short string winding the Milne circle at a fixed time $\left.i r_{0}\right)$.

The action of the operator 2.47) on this test function can not be computed in a closed form, but we can extract the main features by studying the asymptotic behavior using steepest descent methods. First we diagonalize one of the derivative operators, say $\partial_{+}$, by Fourier transform in $x^{+}$,

$$
\begin{aligned}
A(x) & =\frac{1}{2 \pi} \int_{-\infty}^{\infty} d p^{-} e^{-i \Delta p^{-} \partial_{-}} \int_{-\infty}^{\infty} d y^{+} e^{+i p^{-}\left(y^{+}-x^{+}\right)} \exp \left(-\frac{\left(y^{+} x^{-}+r_{0}^{2}\right)^{2}}{a^{2} r_{0}^{2}}\right) \\
& =\frac{a r_{0} \sqrt{\pi}}{2 \pi} \int_{-\infty}^{\infty} d p^{-} e^{-i \Delta p^{-} \partial_{-}} \frac{e^{-i p^{-} x^{+}}}{\left|x^{-}\right|} \exp \left[-p^{-}\left(\frac{r_{0}}{2 x^{-}}\right)^{2}\left(a^{2} p^{-}+4 i x^{-}\right)\right]
\end{aligned}
$$

The action of $e^{-i \Delta p^{-} \partial_{x^{-}}}$is to shift the $x^{-}$coordinate by an amount $-i \Delta p^{-}$. After changing the integration variable to $q=\Delta p^{-} / x^{-}$, we find

$$
A(x)=\frac{a r_{0} \sqrt{\pi}}{2 \pi \Delta} \int_{-\infty}^{\infty} d q \frac{e^{-i \frac{x^{+} x^{-}}{\Delta} q}}{|1-i q|} \exp \left[-\frac{r_{0}^{2}}{4 \Delta} \frac{q}{(1-i q)^{2}}\left(\frac{a^{2}}{\Delta} q+4 i(1-i q)\right)\right]
$$

The integral above is well defined as can be seen by deforming the integration contour into the lower half $q$-plane for $x^{+} x^{-}>0$, i.e. for an observer in the future Milne region. The denominator is written as $|1-i q| \rightarrow \sqrt{1+q^{2}}$, paying the cost of a branch cut on the imaginary axis extending from $-i$ to $-i \infty$, enclosed by the integration contour around (the other branch cut stretching from $+i$ stretching up to $+i \infty$ is not relevant for this region). The integral is dominated by three saddle points whose qualitative behavior changes between small ${ }^{8}$ and large $\Delta / a^{2}$. We will explore these regions separately.

\footnotetext{
${ }^{8}$ Recall, however, that for a fixed boost parameter we can take $\Delta$ to infinity but not to zero, since $\nu$ is bounded from below
} 
For large $\Delta / a^{2}$ (i.e. large stringy fuzziness) the saddle points are (to leading order in $\left.a^{2} / \Delta\right)$ :

$$
q_{1}=-i+i \frac{a^{2}}{2 \Delta} \quad, \quad q_{2,3}=-i \mp \sqrt{\frac{r_{0}^{2}}{x^{+} x^{-}}}
$$

Using these saddle points we can estimate (2.53) in this limit:

$$
A(x)=\sim \frac{a^{3} e^{-\frac{r_{0}^{2}}{a^{2}}-\frac{x^{+} x^{-}}{\Delta}}}{4 \Delta^{\frac{3}{2}}}+\frac{a r_{0} e^{\frac{r_{0}^{2} x^{+} x^{-}}{\Delta}}}{\sqrt{\Delta}\left[x^{+} x^{-}\left(r_{0}^{2}+x^{+} x^{-}\right)\right]^{\frac{1}{4}}} \cos \left(\frac{\Theta}{4}+2 \frac{r_{0} \sqrt{x^{+} x^{-}}}{\Delta}\right)
$$

where $\Theta=\tan ^{-1}\left(2 \sqrt{x^{+} x^{-} / r_{0}^{2}}\right)$. The saddle points are reliable in the limit of large $r_{0}^{2} x^{+} x^{-} / \Delta^{2}$. Furthermore, the main contribution is the second (coming from $q_{2}, q_{3}$ ). The smeared function is therefore concentrated in a region of the past/future wedges around $r_{0}$ with r.m.s. size $\sqrt{\Delta}$, much larger than the original size of order $a$.

For small $\Delta / a^{2}$ instead (i.e. in the semi-classical limit), the saddle points are located, to leading order in $\Delta / a^{2}$, at

$$
q_{1}=-2 i \frac{\Delta}{a^{2}}\left(1+\frac{x^{+} x^{-}}{r_{0}^{2}}\right) \quad, \quad q_{2,3}=-\frac{3}{2} i \mp\left(\frac{a^{2}}{\Delta} \frac{r_{0}^{2}}{2 x^{+} x^{-}}\right)^{1 / 2}
$$

giving a saddle point estimate

$$
A(x) \sim e^{-\frac{\left(x^{+} x^{-}+r_{0}^{2}\right)^{2}}{a^{2} r_{0}^{2}}}+\left(\frac{2 a^{2} r_{0}^{2}}{\Delta x^{+} x^{-}}\right)^{\frac{1}{4}} e^{\frac{a^{2} r_{0}^{2}}{4 \Delta^{2}}} \cos \left(\frac{\pi}{4}+\frac{a r_{0} \sqrt{2 x^{+} x^{-}}}{\Delta^{\frac{3}{2}}}\right)
$$

One recovers in the first term the original test function $A_{0}(x)$, from the contribution of the saddle point $q_{1}$. However, the second term, which comes from the saddle points $q_{2}, q_{3}$, overwhelms the first in the limit $\Delta \rightarrow 0$. Although the saddle points $q_{2,3}$ move off to infinity in this limit, we have not found any indication that they should not contribute to the contour integral. If correct, this implies that the limit $\beta \rightarrow 0$ is singular, against expectations from usual decoupling arguments. Strictly speaking, however, in order to obtain a finite string background we need to take $x^{+} x^{-} \rightarrow \infty$ at the same time as $\beta \rightarrow 0$, holding $x^{+} x^{-} \beta^{2}$ fixed. It would be interesting to investigate this limit in detail.

\section{Scattering amplitudes with two twisted strings}

We now turn to tree-level scattering amplitudes involving two twisted strings, and one or more untwisted states. For this purpose, it is convenient to view the string world-sheet as an infinitely long cylinder, with the twisted vertex operators being inserted at $\tau= \pm \infty$. Amplitudes can then simply be computed by operator methods, in the Hilbert space of the twisted string. In this section, we compute the scattering amplitude of of one tachyon or graviton off a short or a long winding string. We also study the four point function of two twisted and two untwisted strings, and find that it diverges as a result of large winding string emission. 


\subsection{Two-twisted one-untwisted amplitude}

We now turn to the amplitude involving two twisted strings of (identical) winding number $w$, and one untwisted string. We will start by choosing the latter as a tachyon, although our interest eventually lies in the case of a graviton. As before, the twisted strings are assumed to be in their ground state, and on shell. We do not impose the mass-shell condition for the untwisted state at this stage, however.

\subsubsection{Tachyon form factor}

Since the twisted strings are assumed to be on-shell, the amplitude is independent of the location of the untwisted vertex operator, which we choose to be at $\tau=\sigma=0$ (i.e. $z=\bar{z}=1$ ). The amplitude is thus given by

$$
\langle 1|T(2)| 3\rangle=\int_{-\infty}^{\infty} d v_{2}\left\langle 1\left|: e^{i\left(p_{2}^{+}\left(v_{2}\right) X^{-}+p_{2}^{-}\left(v_{2}\right) X^{+}+p_{2}^{i} X^{i}\right)}:\right| 3\right\rangle e^{i j_{2} v_{2}}
$$

where $p_{2}^{ \pm}(v)=e^{\mp v} p_{2}^{ \pm}$. The integral over $v_{2}$ projects the tachyon vertex operator to a boost-invariant state with integer momentum $j_{2}$. It is easy to see that the integral over $v_{2}$ contributes a momentum-conserving Kronecker symbol ${ }^{9} \delta_{-j_{1}+j_{2}+j_{3}}$, where the boost momentum of the twisted states 1 and 3 is given by (2.9). We will therefore set $v_{2}=0$ for now and reinstate momentum conservation at the end of the computation. Similarly, the transverse part yields a delta function enforcing transverse momentum conservation, $\delta\left(-\vec{p}_{1}+\vec{p}_{2}+\vec{p}_{3}\right)$. The amplitude now factorizes as a product of a zero-mode and excitedmode piece,

$$
\left\langle 1\left|e^{i p_{2}^{+} X^{-}+i p_{2}^{-} X^{+}}\right| 3={ }_{\text {out }}\left\langle f_{1}\left|e^{i p_{2}^{+} X_{0}^{-}+i p_{2}^{-} X_{0}^{+}}\right| f_{3}\right\rangle_{\text {in }} \times\left\langle\nu\left|e^{i p_{2}^{+} X_{e x}^{-}+i p_{2}^{-} X_{e x}^{+}}\right| \nu\right\rangle\right.
$$

where the contribution of the excited modes was already computed in (2.36).

We now turn to the zero-mode part. Using the real space representation (2.6), the zero-mode contribution reduces to the overlap

$$
{ }_{\text {out }}\left\langle f_{1}\left|e^{i\left(p_{2}^{+} X_{0}^{-}+p_{2}^{-} X_{0}^{+}\right)}\right| f_{3}\right\rangle_{\text {in }}=\int d x^{+} d x^{-} f_{1}^{*}\left(x^{+}, x^{-}\right) e^{i\left(p_{2}^{+} x^{-}+p_{2}^{-} x^{+}\right)} f_{3}\left(x^{+}, x^{-}\right)
$$

where $f_{1}$ and $f_{3}$ are eigenmodes of the Klein-Gordon equation with electric charge $\nu$. Were it not for the contribution of the excited modes (2.36), the tachyon form factor evaluated at a point $\left(x^{+}, x^{-}\right)$would therefore be equal to the product of the twisted wave functions $f_{1}^{*}\left(x^{+}, x^{-}\right) f_{3}\left(x^{+}, x^{-}\right)$at that point. Instead, as described in Section 2.5, the tachyon form factor will be smeared over a size $\sqrt{\Delta}$.

In order to evaluate the overlap (3.3) for on-shell twisted wave functions, let us use the in and out oscillator representations for $f_{3}$ and $f_{1}$, respectively. Replacing $X_{0}^{ \pm}$by its expression (2.10) in terms of the quasi zero-modes, the amplitude factorizes into a product of expectation values in the left and right-moving zero-mode sectors,

$$
{ }_{\text {out }}\left\langle f_{1}\left|e^{i\left(p_{2}^{+} X_{0}^{-}+p_{2}^{-} X_{0}^{+}\right)}\right| f_{3}\right\rangle_{\text {in }}=e^{\frac{p_{2}^{+} p_{2}^{-}}{i \nu}}\left\langle M_{1}\left|e^{-\frac{i}{\nu} p_{2}^{+} \alpha^{-}} e^{\frac{i}{\nu} p_{2}^{-} \alpha^{+}}\right| M_{3}\right\rangle\left\langle\tilde{M}_{1}\left|e^{-\frac{i}{\nu} p_{2}^{-} \tilde{\alpha}^{+}} e^{+\frac{i}{\nu} p_{2}^{+} \tilde{\alpha}^{-}}\right| \tilde{M}_{3}\right\rangle
$$

\footnotetext{
${ }^{9}$ Switching the boost identification $(2.20)$ from the oscillator variables to $v$ effectively reduces the range of $v$ to the interval $[0,2 \pi \beta]$, leading to a Kronecker symbol rather than a Dirac delta.
} 
where we used the Baker-Campbell-Hausdorff formula to split each exponential into a product of diagonalized operators. Recalling that the inner product in the oscillator representation is given by (2.18), we obtain

$$
\begin{aligned}
\left\langle f_{1}\left|e^{i\left(p_{2}^{+} X_{0}^{-}+p_{2}^{-} X_{0}^{+}\right)}\right| f_{3}\right\rangle=e^{\frac{p_{2}^{+} p_{2}^{-}}{i \nu}} & \int d^{4} \alpha f_{1}^{*}\left(\tilde{\alpha}^{+}, \alpha^{-}\right) \\
& e^{\frac{i}{\nu}\left[\alpha^{+} \alpha^{-}+\tilde{\alpha}^{+} \tilde{\alpha}^{-}+p_{2}^{-}\left(\alpha^{+}-\tilde{\alpha}^{+}\right)-p_{2}^{+}\left(\alpha^{-}-\tilde{\alpha}^{-}\right)\right]} f_{3}\left(\alpha^{+}, \tilde{\alpha}^{-}\right)
\end{aligned}
$$

Representing the in and out wave functions as continuous derivatives with respect to generating parameters as in (2.23), (2.26), the integral over $\alpha^{ \pm}, \tilde{\alpha}^{ \pm}$is Gaussian, leading to

$$
\left\langle f_{1}\left|e^{i\left(p_{2}^{+} X_{0}^{-}+p_{2}^{-} X_{0}^{+}\right)}\right| f_{3}\right\rangle=\left.\nu^{2} \mathcal{D}_{1}^{*} \mathcal{D}_{3} \cdot e^{i \nu\left(\zeta^{+} \zeta^{-}+\tilde{\zeta}^{+} \tilde{\zeta}^{-}\right)-i\left(\zeta^{+}+\tilde{\zeta}^{+}\right) p_{2}^{-}-i\left(\zeta^{-}+\tilde{\zeta}^{-}\right) p_{2}^{+}+\frac{i}{\nu} p_{2}^{+} p_{2}^{-}}\right|_{\zeta^{ \pm}=\tilde{\zeta}^{ \pm}=0}
$$

We may now evaluate the continuous derivatives by using the identity ${ }^{10}$

$$
\begin{aligned}
& {\left.\left[i \frac{\partial}{\partial \zeta^{+}}\right]^{\frac{M_{1}^{2}}{2 i \nu}-\frac{1}{2}}\left[i \frac{\partial}{\partial \zeta^{-}}\right]^{\frac{M_{2}^{2}}{2 i \nu}-\frac{1}{2}} e^{i\left(\nu \zeta^{+} \zeta^{-}-\zeta^{+} p^{-}-\zeta^{-} p^{+}+\frac{1}{\nu} p^{+} p^{-}\right)}\right|_{\zeta^{ \pm}=0}=(i \nu)^{\frac{M_{1}^{2}}{2 i \nu}-\frac{1}{2}}} \\
& \quad \times \Gamma\left(\frac{M_{1}^{2}}{2 i \nu}+\frac{1}{2}\right) \Gamma\left(\frac{M_{2}^{2}}{2 i \nu}+\frac{1}{2}\right)\left(-p^{+}\right)^{\frac{M_{2}^{2}-M_{1}^{2}}{2 i \nu}} U\left(\frac{M_{2}^{2}}{2 i \nu}+\frac{1}{2}, 1+\frac{M_{2}^{2}-M_{1}^{2}}{2 i \nu} ; \frac{i p^{+} p^{-}}{\nu}\right)
\end{aligned}
$$

where $U(\lambda, \mu ; z)$ is Tricomi's confluent hypergeometric function,

$$
U(\lambda, \mu ; z)=\frac{1}{\Gamma(\lambda)} \int_{0}^{\infty} d t t^{\lambda-1}(1+t)^{\mu-\lambda-1} e^{-z t}
$$

Reinstating the momentum-conserving delta functions along the light-cone and the transverse directions, we finally obtain

$$
\begin{aligned}
\left\langle 1\left|: e^{i\left(p_{2}^{+} X^{-}+p_{2}^{-} X^{+}\right)}:\right| 3\right\rangle=g_{s} \delta_{-j_{1}+j_{2}+j_{3}} \delta\left(-\vec{p}_{1}+\vec{p}_{2}+\vec{p}_{3}\right) \exp \left[-p_{2}^{+} p_{2}^{-} \tilde{\Delta}(\nu)\right] \\
(2 \nu)^{-1}\left(-p_{2}^{+}\right)^{\mu-1}\left(-p_{2}^{-}\right)^{\tilde{\mu}-1} U\left(\lambda, \mu, i \frac{p_{2}^{+} p_{2}^{-}}{\nu}\right) U\left(\tilde{\lambda}, \tilde{\mu}, i \frac{p_{2}^{+} p_{2}^{-}}{\nu}\right)
\end{aligned}
$$

where the cumulative effects of the zero and excited mode fluctuations lead to a non-locality parameter

$$
\tilde{\Delta}(\nu) \equiv \Delta(\nu)-\frac{1}{i \nu}=\psi(i \nu)+\psi(1-i \nu)-2 \psi(1)
$$

The parameters of the Whittaker functions appearing in (3.9) are given by

$$
\begin{array}{ll}
\lambda=\frac{1}{2}+\frac{M_{3}^{2}}{2 i \nu} & \tilde{\lambda}=\frac{1}{2}+\frac{\tilde{M}_{3}^{2}}{2 i \nu} \\
\mu=1+\frac{M_{3}^{2}-M_{1}^{2}}{2 i \nu} & \tilde{\mu}=1+\frac{\tilde{M}_{3}^{2}-\tilde{M}_{1}^{2}}{2 i \nu} .
\end{array}
$$

\footnotetext{
${ }^{10}$ This identity follows from using the continuous derivative operator (2.25), or equivalently performing the $\alpha^{ \pm}$integral explicitly, and using the transformation property $U(\lambda, \mu, z)=z^{1-\mu} U(1+\lambda-\mu, 2-\mu, z)$. In particular, even when $M_{1}^{2} /(2 i \nu)-1 / 2=n$ and $M_{2}^{2} /(2 i \nu)-1 / 2=\bar{n}$ are integer, it does not reduce to the analytic continuation of the Euclidean result, which would instead be given by an hypergeometric function $U\left(\mu-\lambda, \mu ;-i p^{+} p^{-} / \nu\right)$, or equivalently by the generalized Laguerre polynomial $L_{\bar{n}}^{n-\bar{n}}\left(-i p^{+} p^{-} / \nu\right)$. Notice that the parameter $\mu$ in 3.8 should not be confused with the two-dimensional squared mass $\mu^{2}$.
} 
In order to compute the real-space representation of the tachyon form factor in the twisted sector, one needs to combine the excited mode contribution (2.36) and the zeromode form evaluated in the previous subsection. The Fourier transform to real space is most conveniently computed from the representation (3.6), which leads to a simple Gaussian integral

$$
\int\left\langle f_{1}\left|V_{T}\left(p_{2}\right)\right| f_{3}\right\rangle e^{-i p_{2} y}=\left.\nu^{2} \mathcal{D}_{1}^{*} \mathcal{D}_{3} \cdot e^{i \nu\left(\zeta^{+} \zeta^{-}+\tilde{\zeta}^{+} \tilde{\zeta}^{-}\right)-\frac{1}{\Delta(\nu)}\left(y^{+}+\zeta^{+}-\tilde{\zeta}^{+}\right)\left(y^{-}+\zeta^{-}-\tilde{\zeta}^{-}\right)}\right|_{\zeta^{ \pm}=\tilde{\zeta}^{ \pm}=0}
$$

Since this expression is still Gaussian in $\zeta$, the continuous derivatives can be computed by employing (3.7) as before. It is interesting to notice that due to the zero-point fluctuations of the string in the twisted sector, the r.m.s. size of the tachyon form factor is now given by $\left\langle\delta y^{+} \delta y^{-}\right\rangle \sim \tilde{\Delta}(\nu)$. In particular, for large winding numbers, the twisted string appears to grow as $\sqrt{\log \nu}$.

We thus conclude that the three-point amplitude involving two twisted strings and one untwisted, all in their ground state with respect to transverse oscillations, is finite, and proportional to the overlap of the zero-mode wave-functions, up to a smearing due to the zero-point fluctuations of the excited modes of the string. Using (3.9) as a source in the equation of motion of the untwisted tachyon, we may further compute the back-reaction of a condensate of winding strings on the tachyon field. We leave this to further work.

\subsubsection{Graviton form factor}

Let us now turn to the case where the untwisted state is a graviton. Using the fact that only zero-modes $\alpha^{ \pm}, \tilde{\alpha}^{ \pm}$have a non-vanishing one-point function in the twisted vacuum, one finds that the scattering amplitude of one graviton and two twisted strings in their ground state is given by the overlap

$$
\begin{aligned}
\left\langle 1\left|T^{ \pm \pm}\right| 3\right\rangle= & \exp \left(-p_{2}^{+} p_{2}^{-} \Delta(\nu)\right) \\
& \left\langle f_{1}\left|\left(\tilde{\alpha}^{ \pm} \mp i \nu p_{2}^{ \pm}+\frac{1}{2} p_{2}^{ \pm}\right) e^{i\left(p_{2}^{+} x^{-}+p_{2}^{-} x^{+}\right)}\left(\alpha^{ \pm} \pm i \nu p_{2}^{ \pm}-\frac{1}{2} p_{2}^{ \pm}\right)\right| f_{3}\right\rangle
\end{aligned}
$$

In this expression, the two signs on $T^{ \pm \pm}$can be chosen independently, and are correlated with the choices of sign for $\alpha^{ \pm}$and $\tilde{\alpha}^{ \pm}$, respectively. The shift by $\pm i \nu p_{2}^{ \pm}$results from the definition of the normal ordered vertex operator in (2.43). The shift by $\pm \frac{1}{2} p_{2}^{ \pm}$on the other hand results from commuting the zero-modes through the plane wave exponential. This choice of ordering is particularly appropriate for the real-space representation, where $\alpha^{ \pm}$are represented by covariant derivatives acting on the charged wave function $f_{3}$, while $\tilde{\alpha}^{ \pm}$act as covariant derivatives on the wave function with opposite charge $f_{1}^{*}$. Altogether, choosing $f_{1}=f_{3}$, we thus find that the graviton one-point function in the twisted vacuum is given by the energy momentum tensor of the complex wave function of the twisted string, smeared under the action of the operator $\exp \left(\Delta(\nu) \partial^{+} \partial^{-}\right)$. This result is in line with the analogy to the charged particle in a constant electric field discussed in [15].

The graviton one-point function for an on-shell twisted state is however more easily computed in the oscillator representation, where all $\alpha^{+}, \tilde{\alpha}^{-}$are moved to the right and 
$\alpha^{-}, \tilde{\alpha}^{+}$and to the left. It is tedious but easy to check that the same rule as in (3.13) applies, namely that $\alpha^{ \pm}$and $\tilde{\alpha}^{ \pm}$must be shifted by $\pm \frac{1}{2} p_{2}^{ \pm}$or $\mp \frac{1}{2} p_{2}^{ \pm}$, depending whether the operator is moved to the left or to the right, respectively. For illustration purposes, we simply give

$$
\begin{aligned}
&\left\langle 1\left|T^{++}\right| 3\right\rangle=-(2 \nu)^{-1} \exp \left[-p_{2}^{+} p_{2}^{-} \Delta(\nu)\right]\left(-p_{2}^{+}\right)^{\mu}\left(-p_{2}^{-}\right)^{\tilde{\mu}-2} \\
& {\left[U\left(\tilde{\lambda}, \tilde{\mu}-1, \frac{i}{\nu} p_{2}^{+} p_{2}^{-}\right)-\frac{1}{2}(1-2 i \nu) p_{2}^{+} p_{2}^{-} U\left(\tilde{\lambda}, \tilde{\mu}, \frac{i}{\nu} p_{2}^{+} p_{2}^{-}\right)\right] } \\
& {\left[U\left(\lambda-1, \mu+1, \frac{i}{\nu} p_{2}^{+} p_{2}^{-}\right)+\frac{1}{2}(1-2 i \nu) U\left(\lambda, \mu, \frac{i}{\nu} p_{2}^{+} p_{2}^{-}\right)\right] }
\end{aligned}
$$

From this result, we may now compute the back-reaction on the metric (and dilaton, KalbRamond fields) of a condensate of twisted strings, by simply inserting $\left\langle 1\left|T^{\mu \nu}\right| 3\right\rangle$ into the equation of motion of the graviton. In momentum space, this amounts to multiplying (3.13) by the graviton propagator in De Donder gauge. We leave the details of this computation to further work.

\subsection{Two-twisted two-untwisted scattering amplitude}

We now come to the scattering amplitude of two twisted (denoted 1,4) and two untwisted (denoted 2,3) on-shell states. We assume as before that the twisted states are in their tachyonic ground state, while the untwisted ones are either both tachyons. By factorizing the amplitude in the limit of small momentum exchange, one expects to recover the off-shell three-point functions computed in the previous section.

By conformal invariance, one may fix the location of the four vertex operators $(1,2,3,4)$ at $\infty, 1, z, 0$, respectively, and obtain the scattering amplitude by integrating over $z$ :

$$
\begin{aligned}
& \langle 1|T(2) T(3)| 4\rangle \equiv \int d z d \bar{z} \int_{-\infty}^{\infty} d v_{2} d v_{3} e^{i\left(j_{2} v_{2}+j_{3} v_{3}\right)} \\
& \times\left\langle 1\left|: e^{i p_{2}^{+}\left(v_{2}\right) X^{-}+i p_{2}^{-}\left(v_{2}\right) X^{+}+p_{2}^{i} X^{i}}:: e^{i p_{3}^{+}\left(v_{3}\right) X^{-}+i p_{3}^{-}\left(v_{3}\right) X^{+}+p_{2}^{i} X^{i}}:\right| 4\right\rangle
\end{aligned}
$$

The integral over $v_{2}, v_{3}$ projects the tachyon vertex operators to orbifold-invariant states with quantized momenta $j_{2}, j_{3}$. As in (3.1), the integral over $v_{2}+v_{3}$ (rendered compact by the identification (2.20)) enforces a momentum-conserving Kronecker symbol $\delta_{-j_{1}+j_{2}+j_{3}+j_{4}}$ which we will reinstate at the end, allowing us to set $v_{2}=0$ in the meantime. The remaining integral over $v_{3} \equiv v$ however is non-trivial, and is expected to be the source of divergences, as in the tree-level four-point amplitudes of untwisted states investigated in Ref. [8].

Just as before, the light-cone contribution to the second line of (3.15) can be computed by operator methods, and factorizes into the product of a zero-mode piece and an excited mode contribution:

$$
{ }_{\text {out }}\left\langle f_{1}\left|e^{i p_{2}^{+} X_{0}^{-}+i p_{2}^{-} X_{0}^{+}} e^{i p_{3}^{+}(v) X_{0}^{-}+i p_{3}^{-}(v) X_{0}^{+}}\right| f_{4}\right\rangle_{i n} \times\left\langle\nu\left|e^{i p_{2}^{+} X_{e x}^{-}+i p_{2}^{-} X_{e x}^{+}} e^{i p_{3}^{+} X_{e x}^{-}+i p_{3}^{-} X_{e x}^{+}}\right| \nu\right\rangle
$$

Let us start with the excited mode piece. As in the three-point function (2.36), the normal ordering of the tachyon vertex operators yields a Gaussian factor $\exp \left[-\left(p_{2}^{+} p_{2}^{-}+p_{3}^{+} p_{3}^{-}\right) \Delta(\nu)\right]$. 
In addition, one needs to commute the positive frequency part of $V_{T}(2)$ with the negative frequency part of $V_{T}(3)$, leading to

$$
\exp \left(-\left[p_{2}^{+} p_{2}^{-}+p_{3}^{+} p_{3}^{-}\right] \Delta(\nu)+2 \Re\left[p_{2}^{-} p_{3}^{+} e^{-v} G(z ; \nu)+p_{2}^{+} p_{3}^{-} e^{v} G(z ;-\nu)\right]\right)
$$

where $G(z ; \nu)$ denotes the propagator in the twisted sector

$$
G(z ; \nu) \equiv \sum_{n=1}^{\infty} \frac{z^{n+i \nu}}{n+i \nu}=\frac{z^{1+i \nu}}{1+i \nu}{ }_{2} F_{1}(1+i \nu, 1 ; 2+i \nu ; z)
$$

In the coincident limit $z \rightarrow 1$,

$$
G(z ; \nu) \rightarrow-\log (1-z)+\psi(1)-\psi(1+i \nu)
$$

recovering the correct short distance singularity.

We now turn to the zero-mode piece. Using the real-space representation, it reduces to the overlap

$$
\int d x^{+} d x^{-} f_{1}^{*}\left(x^{+}, x^{-}\right) e^{i\left(p_{2}^{+} x^{-}+p_{2}^{-} x^{+}\right)} e^{i p_{3}^{+} e^{-v} x^{-}(z, \bar{z})+i p_{3}^{-} e^{v} x^{+}(z, \bar{z})} f_{4}\left(x^{+}, x^{-}\right)
$$

The latter can be computed by the now familiar generating function technique,

$$
\begin{aligned}
& \nu^{2} \mathcal{D}_{1}^{*} \mathcal{D}_{4} \cdot \exp \left[i \nu \left(\zeta^{+} \zeta^{-}\right.\right.\left.+\tilde{\zeta}^{+} \tilde{\zeta}^{-}\right)-i \zeta^{+}\left(p_{2}^{-}+p_{3}^{-} e^{v} z^{-i \nu}\right)-i \zeta^{-}\left(p_{2}^{+}+p_{3}^{+} e^{-v} z^{i \nu}\right) \\
&-i \tilde{\zeta}^{+}\left(p_{2}^{-}+p_{3}^{-} e^{v} \bar{z}^{i \nu}\right)-i \tilde{\zeta}^{-}\left(p_{2}^{+}+p_{3}^{+} e^{-v} \bar{z}^{-i \nu}\right) \\
&\left.\quad+\frac{i}{\nu}\left(p_{2}^{+} p_{2}^{-}+p_{3}^{+} p_{3}^{-}+p_{2}^{+} p_{3}^{-} z^{-i \nu}+p_{2}^{-} p_{3}^{+} \bar{z}^{-i \nu}\right)\right]\left.\right|_{\zeta^{ \pm}=\tilde{\zeta}^{ \pm}=0}
\end{aligned}
$$

Evaluating the continuous derivatives with the help of (3.7), and reinstating the momentumconserving delta-function and the standard contribution of the transverse coordinates $X^{i}$, we finally obtain

$$
\begin{gathered}
\langle 1|T(2) T(3)| 4\rangle=g_{s}^{2}(2 \nu)^{-1} \delta_{-j_{1}+j_{2}+j_{3}+j_{4}} \delta\left(-\vec{p}_{1}+\sum_{i=1}^{3} \vec{p}_{i}\right) \\
\quad \int_{-\infty}^{\infty} d v e^{i j_{3} v} \int d z d \bar{z}|1-z|^{2 \vec{p}_{2} \cdot \vec{p}_{3}}|z|^{2 \vec{p}_{3} \cdot \vec{p}_{4}+\vec{p}_{3} \cdot \vec{p}_{3}-2} \exp \left[-\left(p_{2}^{+} p_{2}^{-}+p_{3}^{+} p_{3}^{-}\right) \tilde{\Delta}(\nu)\right] \\
\exp \left[p_{2}^{-} p_{3}^{+} e^{-v}\left(G(z ; \nu)+G(\bar{z} ;-\nu)+\frac{i}{\nu} \bar{z}^{-i \nu}\right)+p_{2}^{+} p_{3}^{-} e^{v}\left(G(z ;-\nu)+G(\bar{z} ; \nu)+\frac{i}{\nu} z^{-i \nu}\right)\right] \\
{\left[p_{2}^{+}+p_{3}^{+} e^{-v} z^{i \nu}\right]^{\mu-1}\left[p_{2}^{-}+p_{3}^{-} e^{v} \bar{z}^{i \nu}\right]^{\tilde{\mu}-1} U\left(\lambda, \mu, \frac{i}{\nu} A(v, z)\right) U\left(\tilde{\lambda}, \tilde{\mu}, \frac{i}{\nu} \tilde{A}(v, \bar{z})\right)}
\end{gathered}
$$

where $(\lambda, \nu, \tilde{\lambda}, \tilde{\mu})$ are the same as in (3.11) after relabeling the particle 3 into 4 , and we defined

$$
\begin{aligned}
& A(v, z)=\left(p_{2}^{-}+p_{3}^{-} e^{v} z^{-i \nu}\right)\left(p_{2}^{+}+p_{3}^{+} e^{-v} z^{i \nu}\right) \\
& \tilde{A}(v, \bar{z})=\left(p_{2}^{-}+p_{3}^{-} e^{v} \bar{z}^{i \nu}\right)\left(p_{2}^{+}+p_{3}^{+} e^{-v} \bar{z}^{-i \nu}\right)
\end{aligned}
$$


Notice that the correlation function in (3.22) was evaluated for $|z|<1$, but the same result holds when $|z|>1$ due to the transformation properties of hypergeometric functions.

It is easy to check, using (3.19), that the amplitude in the limit $z \rightarrow 1$ correctly factorizes into the product of the three-point amplitude of three untwisted fields with momenta $p_{2}^{ \pm}, p_{3}^{ \pm}, p^{ \pm}=p_{2}^{ \pm}+e^{\mp v} p_{3}^{ \pm}$, and the amplitude $\langle 1|T(p)| 4\rangle$ of two twisted and one untwisted state, respectively:

$$
\langle 1|T(2) T(3)| 4\rangle=g_{s} \int_{-\infty}^{\infty} d v\left\langle 1\left|T\left(p^{ \pm}=p_{2}^{ \pm}+e^{\mp v} p_{3}^{ \pm} ; j_{2}+j_{3} ; \vec{p}_{2}+\vec{p}_{3}\right)\right| 4\right\rangle
$$

where $\langle 1|T(p)| 4\rangle$ is the off-shell three-point function (3.9). One should in principle go to the basis of boost eigenmodes for the intermediate untwisted state, but we will refrain from doing so.

In the limit $z=0$ on the other hand, the amplitude should reduce to a superposition of products of three-point functions $\left\langle 1|T(2)| M^{2}, \tilde{M}^{2}\right\rangle$ and $\left\langle M^{2}, \tilde{M}^{2}|T(3)| 4\right\rangle$, where $\left|M^{2}, \tilde{M}^{2}\right\rangle$ belongs to the continuum of two twisted fields. In order to check that the amplitude factorizes correctly, it important to note that as $z \rightarrow 0$, the limit of $z^{ \pm i \nu}$ is ill-defined. In view of the discussion in [16], we choose to rotate the world-sheet time slightly away from the Euclidean axis, i.e. set $z=e^{i\left(e^{-i \theta} \tau-\sigma\right)}$ and $\bar{z}=e^{i\left(e^{-i \theta} \tau+\sigma\right)}$, respectively, with $\theta$ slightly smaller than $\pi / 2$. According to this prescription, as $\tau \rightarrow-\infty$

$$
z^{n+i \nu} \rightarrow 0 \quad \forall n \geq 1, \quad z^{n-i \nu} \rightarrow 0 \quad \forall n \geq 0
$$

The same holds upon replacing $z$ by $\bar{z}$, since both are related by changing the sign of $\sigma(z$ and $\bar{z}$ are therefore not complex conjugate anymore !). In the limit $\tau \rightarrow-\infty$, the third line of $(3.22)$ can therefore be dropped, leaving simply the overlap of the zero-mode wave functions. By inserting a completeness relation between states 2 and 3 in (3.20), the correct factorization is therefore guaranteed. Nevertheless, it is useful to compute the limit in some detail,

$$
\begin{aligned}
&\langle 1|T(2) T(3)| 4\rangle \rightarrow g_{s}^{2} \delta_{-j_{1}+j_{2}+j_{3}+j_{4} \delta} \delta\left(-\vec{p}_{1}+\sum_{i=1}^{3} \vec{p}_{i}\right) \\
& \int_{-\infty}^{\infty} d v e^{i\left(j_{3}-j_{1}\right) v} \int d z d \bar{z}|z|^{2 \vec{p}_{3} \cdot \vec{p}_{4}+\vec{p}_{3} \cdot \vec{p}_{3}-2} \exp \left[-\left(p_{2}^{+} p_{2}^{-}+p_{3}^{+} p_{3}^{-}\right) \tilde{\Delta}(\nu)\right] \\
&(-1)^{\mu+\tilde{\mu}}\left(p_{2}^{+}\right)^{-\tilde{\lambda}}\left(p_{2}^{-}\right)^{-\lambda}\left(p_{3}^{+}\right)^{\mu-\lambda-1}\left(p_{3}^{-}\right)^{\tilde{\mu}-\tilde{\lambda}-1} z^{-\frac{1}{2} M_{1}^{2}-\frac{i \nu}{2}} \bar{z}^{-\frac{1}{2} \tilde{M}_{1}^{2}-\frac{i \nu}{2}}
\end{aligned}
$$

where we used the asymptotics $U(\lambda, \mu, z) \sim z^{-\lambda}$ as $z \rightarrow \infty$. Notice that, in the limit (3.26), the only dependence in $v$ occurs via an overall exponential factor, and therefore leads to a Dirac delta function $\delta\left(j_{3}-j_{1}\right)$. Since the momenta $j$ are discrete variables, this is strictly infinite whenever $j_{3}=j_{1}$ (and consequently $j_{2}=-j_{4}$ ). Since on-shell twisted states in their tachyonic ground state have $j_{1}=j_{4}=0$, this implies that the intermediate winding state also has $j=0$. We therefore conclude that the four-point amplitude of two twisted states and two untwisted states diverges ${ }^{11}$ due to the propagation of winding

\footnotetext{
${ }^{11}$ Strictly speaking, we cannot rule out a possible cancellation of this singularity coming from another region of the domain of integration.
} 
strings with vanishing boost momentum in the intermediate channel. This result closely parallels the discussion in Ref. [17], where tree-level scattering amplitudes of 4 untwisted states were found to diverge, due to large graviton exchange near the singularity. It would be interesting to see whether the two divergences can somehow be canceled, or whether quantum corrections, for example in the eikonal approximation, lead to a finite amplitude.

\section{Scattering amplitudes with more than two twisted strings}

In the previous section, we have computed scattering amplitudes involving exactly two twisted states, as well an arbitrary number of untwisted states, using Hamiltonian quantization in the twisted vacuum. For more twisted states however, operator techniques are no longer suited, and one should in principle resort to twisted vertex operators. Unfortunately, the conformal theory of twisted vertex operators with a continuous spectrum has not been developed yet. Nevertheless, we shall be able to obtain scattering amplitudes involving three or more twisted states by mapping our problem to an analogue one which has already been solved: the Wess-Zumino-Witten model of a four-dimensional plane wave 21, 22.

\subsection{Misner space and the Nappi-Witten plane wave}

The WZW model based on the non-semi-simple group $H_{4}$, i.e. the three dimensional Heisenberg algebra $[P, \bar{P}]=K$ extended by the generator of rotations in the complex $P$ plane, is known to describe a four-dimensional plane wave, with metric and Neveu-Schwarz flux

$$
d s^{2}=-2 d u d v+d \zeta d \tilde{\zeta}-\frac{1}{4} \zeta \tilde{\zeta} d u^{2}, \quad H=d u d x d \bar{x}
$$

where $\zeta=x_{1}+i x_{2}$ is the complex coordinate in the plane. In the light-cone gauge $u=p \tau$, it is known that the transverse coordinate $X$ has the mode expansion of a complex scalar field twisted by a real angle proportional to the light-cone momentum $p$ [23]. In fact, there exists a free-field representation where the vertex operator of a physical state with nonzero $p$ is just the product of a plane wave along the $(u, v)$ light cone coordinates, times a twist field ${ }^{12}$ creating a cut $z^{p}$ on the world-sheet, where $p$ is in general an irrational number ${ }^{13}$. Correlation functions of physical states have been computed using standard WZW techniques [24, 25], and, by removing the plane wave contribution, it is then possible to extract the correlator of twist fields with arbitrary angle.

For our purposes, it is more convenient to use the current algebra techniques of [24] $]^{14}$. These authors introduce a semi-classical representation for states with non-zero $p$ as

$$
\Phi_{p, q}^{ \pm}=\exp (\mp i p v+i q u) \cdot \exp \left(-\frac{p}{2} \zeta \tilde{\zeta}+i p \zeta x e^{ \pm i u / 2}+i p \tilde{\zeta} \bar{x} e^{ \pm i u / 2}+p x \bar{x} e^{ \pm i u}\right)
$$

where $u, v, \zeta, \tilde{\zeta}$ are the coordinates in the plane wave geometry (4.1), and the variables $x, \bar{x}$ parameterize a representation $V_{p, j}^{ \pm}$of $H_{4}$, with central element $K=i p$ and quadratic

\footnotetext{
${ }^{12}$ For integer $p$, new "spectrally flowed" states appear describing long strings stabilized by the flux [29.

${ }^{13}$ Orbifolds with irrational angle have been recently discussed in [28].

${ }^{14}$ It is also possible to use a Wakimoto-like free-field representation to compute scattering amplitudes of an arbitrary number of twiste fields 25, 26].
} 
Casimir $C=-2 p(j \pm 1 / 2)$. In particular, by expanding in arbitrary powers of $(x, \bar{x})$ one obtains the whole tower of states on which the isometry group $H_{4}$ acts: these states are most conveniently understood as excitations of a two-dimensional harmonic oscillator with frequency $p$. In the conformal field theory, the variables $u, v, \zeta, \tilde{\zeta}$ become fields, and Khnizhnik- Zamolodchikhov equations relate world-sheet derivatives with derivatives with respect to $x, \bar{x}, j, v$, allowing to solve for the conformal blocks.

Our main observation is that the semi-classical vertex (4.2) closely resembles the offshell wave functions (2.14), 2.17) which we introduced to describe twisted strings in the Misner geometry, in particular the generating coordinates $x, \bar{x}$ describing the tower of harmonic oscillator states are quite similar to the continuous generating coordinates $\zeta^{ \pm}, \tilde{\zeta}^{ \pm}$ describing the continuum of states in the inverted harmonic oscillator. To make the connection more precise, notice that the in and out wave functions can be written in the $\left(\tilde{\zeta}^{+}, \zeta^{-}\right)$ and $\left(\zeta^{+}, \tilde{\zeta}^{-}\right)$representations respectively as

$$
\begin{aligned}
f\left(x^{+}, x^{-}\right) & =\mathcal{D}^{i n} \cdot \exp \left(-i \frac{\nu}{2} x^{+} x^{-}-i \nu \tilde{\zeta}^{+} x^{-}-i \nu \zeta^{-} x^{+}-i \nu \tilde{\zeta}^{+} \zeta^{-}\right) \\
f^{*}\left(x^{+}, x^{-}\right) & =\left(\mathcal{D}^{\text {out }}\right)^{*} \cdot \exp \left(-i \frac{\nu}{2} x^{+} x^{+}-i \nu \zeta^{+} x^{-}-i \nu \tilde{\zeta}^{-} x^{+}-i \nu \zeta^{+} \tilde{\zeta}^{-}\right)
\end{aligned}
$$

This is equal to the second factor in $\Phi_{p, q}^{ \pm}$, respectively, upon identifying

$$
\begin{gathered}
p=i \nu, \quad x^{-}=i \tilde{\zeta}, \quad x^{+}=-i \zeta, \\
\zeta^{-}=x e^{i u / 2}, \quad \tilde{\zeta}^{+}=-\bar{x} e^{i u / 2}, \quad \tilde{\zeta}^{-}=\bar{x} e^{-i u / 2}, \quad \zeta^{+}=-x e^{-i u / 2}
\end{gathered}
$$

Finally, the $p=0$ vertex operators

$$
\Phi_{s, j}^{0}=\delta\left(x \bar{x} e^{i u}\right) \exp (i j u) \times \exp \left[\frac{i}{\sqrt{2}} s\left(\zeta \sqrt{\frac{x}{\bar{x}}}+\tilde{\zeta} \sqrt{\frac{\bar{x}}{x}}\right)\right]
$$

agree with the off-shell untwisted wave functions $\exp \left(i p^{+} x^{-}+i p^{-} x^{+}\right)$upon identifying

$$
p^{-}=-\frac{i s}{\sqrt{2}} \sqrt{\frac{x}{\bar{x}}}, \quad p^{+}=\frac{i s}{\sqrt{2}} \sqrt{\frac{\bar{x}}{x}}
$$

Having identified the parameters in the plane wave in terms of those in the Misner space, it is important to stress some differences between the two computations:

- The plane wave computations involves a path integral over the $u$ and $v$ variables, in particular over their respective zero-modes. The integral over $v_{0}$ simply implies conservation of the light-cone momentum $p$, i.e. conservation of the winding number $w$. The integral over $u_{0}$ instead amounts to Fourier transforming the Misner amplitude under the action

$$
\zeta^{ \pm} \rightarrow e^{\mp i u / 2} \zeta^{ \pm}, \quad \tilde{\zeta}^{ \pm} \rightarrow e^{ \pm i u / 2} \tilde{\zeta}^{ \pm}
$$

which therefore needs to be undone if one is to map plane wave amplitude to Misner space. 
- In the plane wave background, setting $x=\bar{x}=0$ gives scattering amplitudes for the ground state of the harmonic oscillator. This is not so for Misner amplitudes, which instead should be obtained by applying the continuous derivative operator (2.25) for arbitrary (complex) order, or equivalently Eq. (3.7). This is the analytic continuation prescription which allows us to obtain physical states of the inverted harmonic oscillator from states of the standard harmonic oscillator.

- The plane wave amplitudes do not involve a projection over boost invariant states. While twisted states, by virtue of the continuous derivation prescription, automatically have a well-defined boost momentum, untwisted vertex operators need to be smeared under the action of the boost.

- Due to the analytic continuation $p \rightarrow i \nu$, the analytic behavior of the Misner amplitudes as a function of $\nu$ is quite different from the plane wave case. For example, the logarithmic growth of the non-locality parameter $\Delta(\nu)$ introduced in (2.36) is replaced by a set of poles at integer $p$ corresponding to the production threshold of long strings [24].

With these words of caution, it is easy to see that the two-point (2.28), three-point (3.6) and four-point (3.21) functions of twisted states in Misner space match the plane wave result.

\subsection{Three-twist amplitude}

We now wish to extract the three-twist amplitude from the plane wave result [24, 25],

$$
\langle 1,2 \mid 3\rangle=\delta_{\mathbb{N}}(L) \delta\left(p_{3}-p_{2}-p_{1}\right)\left|e^{-x_{3}\left(p_{1} x_{1}+p_{2} x_{2}\right)}\left(x_{2}-x_{1}\right)^{L}\right|^{2} \times \frac{1}{\Gamma(1+L)}\left[\frac{\gamma\left(p_{3}\right)}{\gamma\left(p_{1}\right) \gamma\left(p_{2}\right)}\right]^{L}
$$

where $L=q_{3}-q_{1}-q_{2}$ and $\gamma(p) \equiv \Gamma(p) / \Gamma(1-p)$. In this expression, the first terms originate purely from kinematics, while the last term is the OPE coefficient, obtained from factorization of the four-point amplitude ${ }^{15}$. It is interesting to note that the OPE coefficient emerges naturally in the Wakimoto representation as a Virasoro-Shapiro-type integral [25],

$$
\frac{1}{2 \pi} \int d^{2} w|w|^{2\left(p_{1}-1\right)}|1-w|^{2\left(p_{2}-1\right)}=\frac{\gamma\left(p_{1}\right) \gamma\left(p_{2}\right)}{\gamma\left(p_{1}+p_{2}\right)}
$$

hence inherits the usual Regge and Gross-Mende behavior as a function of the Mandelstamlike variables $(s, t, u) \sim\left(p_{1}, p_{2}, p_{3}\right)$.

Our first task is to reproduce the kinematics from the overlap of three twisted off-shell wave functions in the $\zeta^{ \pm}, \tilde{\zeta}^{ \pm}$representation:

$$
\int d x^{+} d x^{-}\left[f_{1} f_{2}\right]^{*} f_{3}=e^{-i \zeta_{3}^{-}\left(\nu_{1} \zeta_{1}^{+}+\nu_{2} \zeta_{2}^{+}\right)-i \tilde{\zeta}_{3}^{+}\left(\nu_{1} \tilde{\zeta}_{1}^{-}+\nu_{2} \tilde{\zeta}_{2}^{-}\right)} e^{-\frac{i \nu_{1} \nu_{2}}{\nu_{3}}\left(\zeta_{1}^{+}-\zeta_{2}^{+}\right)\left(\tilde{\zeta}_{1}^{-}-\tilde{\zeta}_{2}^{-}\right)}
$$

\footnotetext{
${ }^{15}$ The $1 / 2$ in the exponent [24], eq. 4.0.27 can be reabsorbed in the normalization of the vertices [26].
} 
with $\nu_{1}+\nu_{2}=\nu_{3}$. After substituting the plane wave variables, only the last term in the exponential becomes $u$-dependent; the integral over the zero-mode of $u$ leads to

$$
\int_{-\infty}^{\infty} d u_{0} e^{-i L u_{0}} e^{-\frac{i \nu_{1} \nu_{2}}{\nu_{3}}\left(\zeta_{1}^{+}-\zeta_{2}^{+}\right)\left(\tilde{\zeta}_{1}^{-}-\tilde{\zeta}_{2}^{-}\right) e^{i u_{0}}}=\frac{1}{\Gamma(1+L)}\left[\frac{\nu_{1} \nu_{2}}{\nu_{3}}\left(\zeta_{1}^{+}-\zeta_{2}^{+}\right)\left(\tilde{\zeta}_{1}^{-}-\tilde{\zeta}_{2}^{-}\right)\right]^{L}
$$

This reproduces the $\left(x_{2}-x_{1}\right)^{L}$ term in (4.10), as well as the small $p$ behavior of the OPE coefficient, since

$$
\frac{\gamma\left(p_{3}\right)}{\gamma\left(p_{1}\right) \gamma\left(p_{2}\right)} \sim \frac{p_{1} p_{2}}{p_{3}}
$$

Reversing the argument, we find that the complete 3 -twist amplitude in Misner space is given by the generating function

$$
\begin{array}{r}
\langle 1,2 \mid 3\rangle=\left(\nu_{1} \nu_{2} \nu_{3}\right) \delta\left(\nu_{1}+\nu_{2}-\nu_{3}\right) \mathcal{D}_{1}^{*} \mathcal{D}_{2}^{*} \mathcal{D}_{3} \cdot \exp \left[-i \zeta_{3}^{-}\left(\nu_{1} \zeta_{1}^{+}+\nu_{2} \zeta_{2}^{+}\right)-i \tilde{\zeta}_{3}^{+}\left(\nu_{1} \tilde{\zeta}_{1}^{-}+\nu_{2} \tilde{\zeta}_{2}^{-}\right)\right. \\
\left.+\frac{\gamma\left(i \nu_{3}\right)}{\gamma\left(i \nu_{1}\right) \gamma\left(i \nu_{2}\right)}\left(\zeta_{1}^{+}-\zeta_{2}^{+}\right)\left(\tilde{\zeta}_{1}^{-}-\tilde{\zeta}_{2}^{-}\right)\right]
\end{array}
$$

We can now revert to the real space representation, and find that, instead of taking place at a single point $x_{1}^{ \pm}=x_{2}^{ \pm}=x_{3}^{ \pm}$as in (4.12), the interaction is in fact spread with a kernel

$$
\int d x_{1}^{ \pm} d x_{2}^{ \pm}\left[f_{1}\left(x_{1}^{ \pm}\right) f_{2}\left(x_{2}^{ \pm}\right)\right]^{*} \exp \left[\left(x_{1}^{+}-x_{2}^{+}\right)\left(x_{1}^{-}-x_{2}^{-}\right) \Xi\left(\nu_{1}, \nu_{2}\right)\right] f_{3}\left(x_{3}^{ \pm}-\frac{\nu_{1} x_{1}^{ \pm}+\nu_{2} x_{2}^{ \pm}}{\nu_{1}+\nu_{2}}\right)
$$

where the size of the non-locality is given by the (real) ratio

$$
\Xi\left(\nu_{1}, \nu_{2}\right)=-i \frac{1-\frac{i \nu_{3}}{\nu_{1} \nu_{2}} \frac{\gamma\left(i \nu_{3}\right)}{\gamma\left(i \nu_{1}\right) \gamma\left(i \nu_{2}\right)}}{1+\frac{i \nu_{3}}{\nu_{1} \nu_{2}} \frac{\gamma\left(i \nu_{3}\right)}{\gamma\left(i \nu_{1}\right) \gamma\left(i \nu_{2}\right)}}
$$

As $\nu_{i} \rightarrow 0, \Xi\left(\nu_{1}, \nu_{2}\right) \sim 1 /\left(2 \zeta(3) \nu_{3}^{2}\right)$ so that the interaction becomes local. For larger $\nu$ however, the non-locality scale $1 / \sqrt{\Xi}$ diverges when $\nu_{1} \nu_{2} \gamma\left(i \nu_{1}\right) \gamma\left(i \nu_{2}\right)=i \nu_{3} \gamma\left(i \nu_{3}\right)$, before switching sign and vanishing again when $\nu_{1} \nu_{2} \gamma\left(i \nu_{1}\right) \gamma\left(i \nu_{2}\right)=-i \nu_{3} \gamma\left(i \nu_{3}\right)$ (e.g, for $\nu_{1}=\nu_{2}$ this is found numerically to happen at $\nu_{1} \sim 1.236$ and $\nu \sim 3.039$, respectively). It would be interesting to understand the physical origin of this diverging non-locality scale.

We conclude that scattering amplitudes involving 3 or more twist fields can be computed from scattering amplitudes in the Nappi-Witten plane wave by a simple analytic continuation (and a possibly large number of Fourier transforms). It would be interesting to develop the conformal field theory of continuous Lorentzian twist fields, and obtain a Wakimoto-type representation for computing scattering amplitudes including both twisted and untwisted states. This result opens the way to compute the back-reaction of a condensate of twisted states on the twisted fields themselves: deforming the world-sheet action with $\lambda V_{w}+\bar{\lambda} V_{-w}$, it is necessary to include a deformation by $V_{ \pm 2 w}$ at order $|\lambda|^{2}$ to preserve conformal invariance. We leave a more detailed analysis for future work.

Acknowledgments: The authors are happy to thank G. d'Appollonio, Y. Cheung, E. Kiritsis, D. Kutasov, G. Moore, E. Rabinovici, M. Rozali, K. Savvidy for useful discussions. B.P. 
is grateful to the Weizmann Institute for hospitality during part of this project, and to Rochelle and Phalevi for their patience during the last stages of this research. M.B. is grateful to Ecole Polytechnique and to the Erwin Schrödinger Institute for their hospitality during part of this work. The work of M.B. is supported by Israeli Academy of Science centers of excellence program, by the Minerva Foundation, by EEC RTN-2000-001122 and by the Einstein Center for Theoretical Physics.

\section{Appendix}

\section{A. Scattering amplitudes of untwisted strings}

Tree-level scattering amplitudes for untwisted states for string theory in Misner space have been investigated in [17], revealing divergences from a kinematical regime governed by the Regge limit of the standard Virasoro-Shapiro amplitude. The divergences can be traced back to large graviton exchange near the singularity, as the particles experience a high blue-shfit. The authors of [7] have demonstrated that similar divergences in the parabolic orbifold can be regularized by deforming the background to the null-brane spacetime [30, 31] (i.e. combining the null boost with a translation, much as Grant space is a deformation of the Misner space): in this deformed background, wave-packets with a continuous momentum distribution, except for remaining collinear divergences [7]. In this Appendix, we perform a similar analysis for tree-level scattering amplitudes of untwisted states in Grant space. We find that, unlike the null-brane case, divergences remain even for non-zero momentum transfer, although the range of dangerous momenta is reduced with respect to Misner space.

\section{A.1 Four-graviton amplitude in type II superstring}

In order to avoid tachyon-related poles, we consider the type II superstring on Grant space, i.e. the orbifold of Minkowski space $\mathbb{R}^{1,2}$ by the orbifold action

$$
\left(x^{+}, x^{-}, x_{2}\right) \rightarrow\left(e^{2 \pi \beta} x^{+}, e^{-2 \pi \beta} x^{-}, x_{2}+2 \pi R\right)
$$

The vertex operator for NS-NS massless states is given, in the $(-1,-1)$ superghost picture, by

$$
\mathcal{V}^{-1,-1}=g_{s} \int d^{2} z \int_{-\infty}^{\infty} d u \zeta_{\mu \nu}: e^{-\phi-\tilde{\phi}} \psi^{\mu} \tilde{\psi}^{\nu} e^{i P \cdot X}:
$$

where $P=\left(e^{-u} p^{+}, e^{u} p^{-}, p, \vec{p}\right)$ is the momentum on the covering space, and $\zeta_{\mu \nu}$ a polarization tensor (which also depends on $j, u$ ). Invariance under the orbifold group imposes the condition

$$
p=\frac{n-\beta j}{R} \quad, \quad n \in \mathbb{Z} \quad, \quad p, j \in \mathbb{R} .
$$

We also use the notations of [17] for the two-dimensional mass:

$$
m^{2} \equiv 2 p^{-} p^{+}=\vec{p}^{2}+p^{2}
$$


As in [17], the tree-level scattering amplitude of untwisted states is obtained by smearing the standard scattering amplitude for four massless NS-NS fiels in flat space [32] over the action of the boosts,

$$
\begin{aligned}
\mathcal{A}_{4 N S}=-i \pi^{2} g_{s} \alpha^{\prime 3} R^{4}(2 \pi)^{11} \delta^{7} & \left(\sum_{i} \vec{p}_{i}\right) \delta\left(\sum_{i} n_{i}\right) \delta\left(\sum_{i} j_{i}\right) \\
& \cdot \int_{0}^{\infty} d v_{4} \hat{K} H(s) H(t) H(u) \frac{v_{2}^{-i j_{2}+1} v_{3}^{-i j_{3}+1} v_{4}^{-i j_{4}-1}}{\left|p_{2}^{+} p_{3}^{-} v_{3}^{2}-p_{3}^{+} p_{2}^{-} v_{2}^{2}\right|}
\end{aligned}
$$

where $s, t, u$ denote the Mandelstam variables of the boosted particles, e.g.

$$
s=2\left(p_{1}^{+} p_{2}^{-} v_{2}+p_{1}^{-} p_{2}^{+} \frac{1}{v_{2}}\right)-2 p_{1} p_{2}-2 \overrightarrow{p_{1}} \cdot \overrightarrow{p_{2}}
$$

the function $H(x)==\Gamma\left(-\frac{\alpha^{\prime}}{4} x\right) / \Gamma\left(1+\frac{\alpha^{\prime}}{4} x\right)$ and the kinematical factor

$$
\hat{K}=t_{\nu 1 \ldots \nu_{4}}^{\mu_{1} \ldots \mu_{4}} t_{\sigma_{1} \ldots \sigma_{4}} \prod_{r=1}^{4} \zeta_{\mu_{r} \rho_{r}}^{r} P_{r}^{\nu_{r}} P_{r}^{\sigma_{r}} .
$$

In this expression, the $P_{i}$ 's are the covering-space momenta, and the $t^{8}$ tensors can be found in [32]. Using momentum conservation on the covering space, two of the variables variables $\left(v_{2}, v_{3}\right)$ have been written as the positive solution(s) of the following system ${ }^{16}$ :

$$
\left\{\begin{array}{l}
p_{1}^{-}+p_{2}^{-} v_{2}+p_{3}^{-} v_{3}+p_{4}^{-} v_{4}=0 \\
p_{1}^{+}+p_{2}^{+} \frac{1}{v_{2}}+p_{3}^{+} \frac{1}{v_{3}}+p_{4}^{+} \frac{1}{v_{4}}=0
\end{array}\right.
$$

The limit of $v_{4} \rightarrow \infty$ is the familiar Regge limit ${ }^{17}$. The solutions for $v_{2}, v_{3}$ to the leading order in $v_{4}$ are:

$$
\begin{array}{lll}
p_{4}^{+} p_{2}^{+}<0 \quad, \quad p_{3}^{+} p_{1}^{+}<0 \quad \Rightarrow \quad v_{2}=-\frac{p_{4}^{-}}{p_{2}^{-}} v_{4} \quad, \quad v_{3}=-\frac{p_{3}^{+}}{p_{1}^{+}} \\
p_{4}^{+} p_{3}^{+}<0 \quad, \quad p_{2}^{+} p_{1}^{+}<0 \quad \Rightarrow \quad v_{2}=-\frac{p_{2}^{+}}{p_{1}^{+}} \quad, \quad v_{3}=-\frac{p_{4}^{-}}{p_{3}^{-}} v_{4}
\end{array}
$$

We focus on the first solution where $s \sim-2 p_{1}^{+} p_{4}^{-} v_{4}$ is large and $t$ is finite. The contribution to the amplitude from this range is:

$$
\begin{aligned}
& \mathcal{A}_{4 N S} \sim-\frac{i \pi^{2} g_{s} \alpha^{\prime 4}}{2} R^{4}(2 \pi)^{11} \delta^{7}\left(\sum_{i} \vec{p}_{i}\right) \delta\left(\sum_{i} n_{i}\right) \delta\left(\sum_{i} j_{i}\right) \hat{K}_{4} \\
& \quad \times \quad \int_{\Lambda \gg 1}^{\infty} d \sigma\left(-\frac{p_{4}^{-}}{p_{2}^{-}}\right)^{-i j_{2}}\left(-\frac{p_{3}^{+}}{p_{1}^{+}}\right)^{-i j_{3}}\left|-\frac{\alpha^{\prime}}{2} p_{1}^{+} p_{4}^{-}\right|^{1+\frac{\alpha^{\prime} t}{2}} \frac{\Gamma\left(-\frac{\alpha^{\prime} t}{4}\right)}{\Gamma\left(1+\frac{\alpha^{\prime} t}{4}\right)} e^{\left(1+\frac{\alpha^{\prime} t}{2}+i j_{1}+i j_{3}\right) \sigma}
\end{aligned}
$$

\footnotetext{
${ }^{16}$ The $v_{i}$ 's are defined from the original $u_{i}$ 's by the change of variables: $v_{i} \equiv e^{u_{1}-u_{i}}$.

${ }^{17}$ Note that the $v_{4} \rightarrow 0$ limit gives similar results when evaluated to leading order in $1 / v_{4}$, switching the role of $p_{i}^{+} \leftrightarrow p_{i}^{-}$and $v_{i} \leftrightarrow 1 / v_{i}$.
} 
where $\hat{K}_{4}$ is the leading term in $\hat{K}$ as $v_{4} \rightarrow \infty$,

$$
\hat{K}=\hat{K}_{4} v_{4}^{4}+O\left(v_{4}^{3}\right)
$$

For the superstring case $t$ is strictly negative, so we find divergences within the kinematical regime:

$$
-t=\left(\vec{p}_{1}+\vec{p}_{3}\right)^{2}+\left(\frac{\left(n_{1}+n_{3}\right)-\beta\left(j_{1}+j_{3}\right)}{R}\right)^{2} \leqslant \frac{2}{\alpha^{\prime}}
$$

generalizing the result obtained for the bosonic string in Misner space in [17.

\section{A.2 Scattering amplitudes for wave-packets in Grant space}

As an attempt to remove the divergences found in the four-point amplitudes we follow the procedure of [7], and consider wave-packets constructed out of a superposition of wavefunctions with different momenta $j$. Let us start from wave functions with a well-defined value of $(j, \vec{p})$ [14]:

$$
\Psi_{n, j, m, \epsilon^{ \pm}, \vec{p}}\left(X^{+}, X^{-}, X, \vec{X}\right)=\sqrt{\frac{m}{2 \pi R}} e^{i \vec{p} \cdot \vec{X}+i \frac{n-\beta j}{R} X} \int_{-\infty}^{\infty} d u e^{-i \epsilon^{-} \frac{m}{\sqrt{2}} X^{+} e^{u}-i \epsilon^{+} \frac{m}{\sqrt{2}} X^{-} e^{-u}-i j u}
$$

where $m=\sqrt{2\left|p^{+} p^{-}\right|}>0$ and $\left(\epsilon^{+}\right)^{2}=\left(\epsilon^{-}\right)^{2}=1$. The wave-functions (A.13) form a complete basis in Grant space, however they are badly behaved near the light-cone $\left(X^{+} X^{-}=0\right)$ :

$$
\Psi_{n, j, m, \epsilon^{ \pm}, \vec{p}} \sim\left[\frac{1-\operatorname{coth} \pi j}{\Gamma(1+i j)}\left(\frac{m}{\sqrt{2}}\left|X^{+}\right|\right)^{i j}-\frac{\operatorname{csch} \pi j}{\Gamma(1-i j)}\left(\frac{m}{\sqrt{2}}\left|X^{+}\right|\right)^{-i j}\right]+O\left(X^{+}, X^{-}\right)
$$

In the latter formula we chose explicitly $\epsilon^{+} X^{-}, \epsilon^{-} X^{+}>0$. The wave functions for which we can expect the best behavior are the ones that vanish near the light cone. For this, we consider wave-packets

$$
\int \operatorname{dj} F(j) \Psi_{n, j, m, \epsilon^{ \pm}, \vec{p}}
$$

such that

$$
\int_{-\infty}^{\infty} d j F(j) x^{i j}=\tilde{F}(\log x) \stackrel{x \rightarrow 0}{\longrightarrow} 0
$$

where $\tilde{F}$ is the Fourier transform of $F$. In the Regge limit, the four-point amplitude for such wave packets is given by

$$
\begin{aligned}
& \prod_{i=1}^{4}\left(\int_{-\infty}^{\infty} d j_{i} F^{(i)}\left(j_{i}\right)\right) \mathcal{A}_{4 N S}\left(j_{1}, j_{2}, j_{3}, j_{4}\right)= \\
& \quad=\int d j_{1} d j_{2} d j d \tilde{j} F^{(1)}\left(j_{1}\right) F^{(2)}\left(j_{2}\right) F^{(3)}\left(j-j_{1}\right) F^{(4)}\left(\tilde{j}-j_{2}\right) \mathcal{A}_{4 N S}\left(j_{1}, j_{2}, j-j_{1}, \tilde{j}-j_{2}\right)
\end{aligned}
$$


The integration over $j$ amounts to a Fourier transform evaluated at $\sigma \rightarrow \infty$. Thus the amplitude can be evaluated as ${ }^{18}$ :

$$
\begin{aligned}
& \prod_{i=1}^{4}\left(\int_{-\infty}^{\infty} d j_{i} F^{(i)}\left(j_{i}\right)\right) \mathcal{A}_{4 N S}\left(j_{1}, j_{2}, j_{3}, j_{4}\right)= \\
& \delta^{(7)}\left(\sum_{i} p_{i}\right) \delta\left(\sum_{i} n_{i}\right) \hat{K}_{4}\left|-\frac{\alpha^{\prime}}{2} p_{1}^{+} p_{4}^{-}\right|^{1-\frac{\alpha^{\prime}}{2} \vec{p}_{t}^{2}} \int_{\Lambda \gg 1}^{\infty} d \sigma e^{\sigma\left[1-\frac{\alpha^{\prime}}{2} \vec{p}_{t}^{2}\right]} \\
& {\left[\left(\widetilde{F}^{(2)} \widetilde{F}^{(4)}(x)\right) *\left(\widetilde{F}^{(1)} \widetilde{F}^{(3)}(x)\right) *\left(\frac{1}{\sqrt{\sigma}} e^{-\frac{x\left(x-i 2 \alpha^{\prime} \frac{n}{R} E \sigma\right)}{4 E^{2} \sigma}}\right) *\left(e^{\left.i \frac{n}{\beta} x-|x| \sqrt{\frac{\vec{p}_{t}^{2}}{E^{2}}}\right)}\right]_{x=\sigma}\right.}
\end{aligned}
$$

where $E=\beta / R, \vec{p}_{t}=p_{1}+p_{3}, n=n_{1}+n_{3}$, and $*$ denotes the convolution product (i.e. multiplication of the Fourier transforms).

At large $\sigma$ the convolution is dominated by the slowest decaying function. If all the Fourier transforms $\widetilde{F}^{(i)}(x)$ go to zero at $|x| \rightarrow \infty$ faster then $e^{-|x| \sqrt{\vec{p}_{t}^{2} / E^{2}}}$ the amplitude A.18) behaves at the Regge limit as:

$$
\sim \delta^{(7)}\left(\sum_{i} p_{i}\right) \delta\left(\sum_{i} n_{i}\right) \hat{K}_{4}\left|-\frac{\alpha^{\prime}}{2} p_{1}^{+} p_{4}^{-}\right|^{1-\frac{\alpha^{\prime}}{2} \vec{p}_{t}^{2}} \int_{\Lambda \gg 1}^{\infty} d \sigma \exp \left[\sigma\left(1-\frac{\alpha^{\prime}}{2} \vec{p}_{t}^{2}-\sqrt{\frac{\vec{p}_{t}^{2}}{E^{2}}}\right)\right] .
$$

Alas, the range of divergences from (A.12) is reduced but not removed:

$$
\left(\vec{p}_{1}+\vec{p}_{3}\right)^{2} \leq \frac{\left(\sqrt{1+2 \alpha^{\prime} E^{2}}-1\right)^{2}}{\left(\alpha^{\prime} E\right)^{2}}, \quad E=\frac{\beta}{R}
$$

As $R \rightarrow 0$, this reduces to $(\mathrm{A} .12$ as expected.

\section{References}

[1] C. W. Misner, in Relativity Theory and Astrophysics I: Relativity and Cosmology, edited by J. Ehlers, Lectures in Applied Mathematics, Vol. 8 (American Mathematical Society, Providence, 1967), p. 160.

[2] G. T. Horowitz and A. R. Steif, "Singular String Solutions With Nonsingular Initial Data," Phys. Lett. B 258, 91 (1991).

[3] N. Seiberg, "From big crunch to big bang - is it possible?," arXiv:hep-th/0201039.

[4] C. R. Nappi and E. Witten, "A Closed, expanding universe in string theory," Phys. Lett. B 293, 309 (1992) [arXiv:hep-th/9206078].

[5] S. Elitzur, A. Giveon, D. Kutasov and E. Rabinovici, "From big bang to big crunch and beyond," JHEP 0206, 017 (2002) [arXiv:hep-th/0204189]; S. Elitzur, A. Giveon and E. Rabinovici, "Removing singularities," JHEP 0301, 017 (2003) [arXiv:hep-th/0212242].

\footnotetext{
${ }^{18}$ With the hindsight that the divergences arise from $\left|\alpha^{\prime} t\right| \leq 2$, we expand the $\Gamma$ functions near $t=0$.
} 
[6] H. Liu, G. Moore and N. Seiberg, "Strings in a time-dependent orbifold," JHEP 0206, 045 (2002) [arXiv:hep-th/0204168];

[7] H. Liu, G. Moore and N. Seiberg, "Strings in time-dependent orbifolds," JHEP 0210, 031 (2002) [arXiv:hep-th/0206182].

[8] B. Craps, D. Kutasov and G. Rajesh, "String propagation in the presence of cosmological singularities," JHEP 0206, 053 (2002) [arXiv:hep-th/0205101]; B. Craps and B. A. Ovrut, "Global fluctuation spectra in big crunch / big bang string vacua," arXiv:hep-th/0308057.

[9] V. Balasubramanian, S. F. Hassan, E. Keski-Vakkuri and A. Naqvi, "A space-time orbifold: A toy model for a cosmological singularity," Phys. Rev. D 67 (2003) 026003 [arXiv:hep-th/0202187]; R. Biswas, E. Keski-Vakkuri, R. G. Leigh, S. Nowling and E. Sharpe, "The taming of closed time-like curves," JHEP 0401 (2004) 064 [arXiv:hep-th/0304241].

[10] C. V. Johnson and H. G. Svendsen, "An exact string theory model of closed time-like curves and cosmological singularities," arXiv:hep-th/0405141.

[11] J. D. Grant, "Cosmic strings and chronology protection," Phys. Rev. D 47 (1993) 2388 [arXiv:hep-th/9209102].

[12] M. Banados, C. Teitelboim and J. Zanelli, "The Black Hole In Three-Dimensional Space-Time," Phys. Rev. Lett. 69 (1992) 1849 [arXiv:hep-th/9204099].

[13] L. Cornalba and M. S. Costa, "A New Cosmological Scenario in String Theory," Phys. Rev. D 66, 066001 (2002) [arXiv:hep-th/0203031]; L. Cornalba, M. S. Costa and C. Kounnas, "A resolution of the cosmological singularity with orientifolds," Nucl. Phys. B 637, 378 (2002) [arXiv:hep-th/0204261]; L. Cornalba and M. S. Costa, "Time-dependent orbifolds and string cosmology," Fortsch. Phys. 52, 145 (2004) [arXiv:hep-th/0310099].

[14] N. A. Nekrasov, "Milne universe, tachyons, and quantum group," arXiv:hep-th/0203112.

[15] M. Berkooz, and B. Pioline, "Strings in an electric field, and the Milne universe," JCAP 0311 (2003) 007 [arXiv:hep-th/0307280].

[16] M. Berkooz, B. Pioline and M. Rozali, "Closed strings in Misner space: Cosmological production of winding strings," JCAP 07 (2004) 003 [arXiv:hep-th/0405126].

[17] M. Berkooz, B. Craps, D. Kutasov and G. Rajesh, "Comments on cosmological singularities in string theory," arXiv:hep-th/0212215.

[18] G. T. Horowitz and J. Polchinski, "Instability of spacelike and null orbifold singularities," Phys. Rev. D 66, 103512 (2002) [arXiv:hep-th/0206228].

[19] M. Karliner, I. R. Klebanov and L. Susskind, "Size And Shape Of Strings," Int. J. Mod. Phys. A 3 (1988) 1981.

[20] D. Bigatti and L. Susskind, "Magnetic fields, branes and noncommutative geometry," Phys. Rev. D 62 (2000) 066004 [arXiv:hep-th/9908056].

[21] C. R. Nappi and E. Witten, "A WZW model based on a nonsemisimple group," Phys. Rev. Lett. 71, 3751 (1993) [arXiv:hep-th/9310112].

[22] D. I. Olive, E. Rabinovici and A. Schwimmer, "A Class of string backgrounds as a semiclassical limit of WZW models," Phys. Lett. B 321 (1994) 361 [arXiv:hep-th/9311081]. 
[23] E. Kiritsis and C. Kounnas, Phys. Lett. B 320 (1994) 264 [Addendum-ibid. B 325 (1994) 536] [arXiv:hep-th/9310202]; E. Kiritsis, C. Kounnas and D. Lust, "Superstring gravitational wave backgrounds with space-time supersymmetry," Phys. Lett. B 331, 321 (1994) [arXiv:hep-th/9404114].

[24] G. D'Appollonio and E. Kiritsis, "String interactions in gravitational wave backgrounds," Nucl. Phys. B 674 (2003) 80 [arXiv:hep-th/0305081].

[25] Y. K. Cheung, L. Freidel and K. Savvidy, "Strings in gravimagnetic fields," JHEP 0402 (2004) 054 [arXiv:hep-th/0309005].

[26] M. Bianchi, G. D'Appollonio, E. Kiritsis and O. Zapata, "String amplitudes in the Hpp-wave limit of AdS(3) x S**3," JHEP 0404 (2004) 074 [arXiv:hep-th/0402004].

[27] S. Groot Nibbelink and M. Laidlaw, "Stringy profiles of gauge field tadpoles near orbifold singularities. I: Heterotic string calculations," JHEP 0401 (2004) 004 [arXiv:hep-th/0311013].

[28] D. Kutasov, J. Marklof and G. W. Moore, "Melvin Models and Diophantine Approximation," arXiv:hep-th/0407150.

[29] E. Kiritsis and B. Pioline, "Strings in homogeneous gravitational waves and null holography," JHEP 0208, 048 (2002) [arXiv:hep-th/0204004].

[30] J. Figueroa-O'Farrill and J. Simon, "Generalized supersymmetric fluxbranes," JHEP 0112 (2001) 011 [arXiv:hep-th/0110170].

[31] J. Simon, "The geometry of null rotation identifications," JHEP 0206 (2002) 001 [arXiv:hep-th/0203201].

[32] J. H. Schwarz, "Superstring Theory," Phys. Rept. 89 (1982) 223. 